C. Bandle · J. v. Below · W. Reichel

\title{
Positivity and anti-maximum principles for elliptic operators with mixed boundary conditions
}

Received May 19, 2006

\begin{abstract}
We consider linear elliptic equations $-\Delta u+q(x) u=\lambda u+f$ in bounded Lipschitz domains $D \subset \mathbb{R}^{N}$ with mixed boundary conditions $\partial u / \partial n=\sigma(x) \lambda u+g$ on $\partial D$. The main feature of this boundary value problem is the appearance of $\lambda$ both in the equation and in the boundary condition. In general we make no assumption on the sign of the coefficient $\sigma(x)$. We study positivity principles and anti-maximum principles. One of our main results states that if $\sigma$ is somewhere negative, $q \geq 0$ and $\int_{D} q(x) d x>0$ then there exist two eigenvalues $\lambda_{-1}, \lambda_{1}$ such the positivity principle holds for $\lambda \in\left(\lambda_{-1}, \lambda_{1}\right)$ and the anti-maximum principle holds if $\lambda \in\left(\lambda_{1}, \lambda_{1}+\delta\right)$ or $\lambda \in\left(\lambda_{-1}-\epsilon, \lambda_{-1}\right)$. A similar, but more complicated result holds if $q \equiv 0$. This is due to the fact that $\lambda_{0}=0$ becomes an eigenvalue in this case and that $\lambda_{1}(\sigma)$ as a function of $\sigma$ connects to $\lambda_{-1}(\sigma)$ when the mean value of $\sigma$ crosses the value $\sigma_{0}=-|D| /|\partial D|$. In dimension $N=1$ we determine the optimal $\lambda$-interval such that the anti-maximum principles holds uniformly for all right-hand sides $f, g \geq 0$. Finally, we apply our result to the problem $-\Delta u+q(x) u=\alpha u+f$ in $D, \partial u / \partial n=\beta u+g$ on $\partial D$ with constant coefficients $\alpha, \beta \in \mathbb{R}$.
\end{abstract}

Keywords. Positivity principle, anti-maximum principle, eigenvalues, Harnack inequality

\section{Introduction}

Let $D \subset \mathbb{R}^{N}$ be a bounded domain with Lipschitz boundary $\partial D$, and let $n$ denote its outer unit normal. This paper deals with boundary value problems of the form

$$
-\Delta u+q(x) u=\lambda u+f \quad \text { in } D, \quad u_{n}=\sigma(x) \lambda u+g \quad \text { on } \partial D,
$$

where $f \in L^{2}(D), g \in L^{2}(\partial D)$. Here $q$ is a bounded, positive function defined on $D$, $\sigma$ is a continuous function defined on $\partial D$ and $\lambda \in \mathbb{R}$ a real parameter. The main feature of this boundary value problem is the appearance of $\lambda$ both in the differential equation and in the boundary condition. Moreover, we make no assumption on the sign of the coefficient $\sigma(x)$.

C. Bandle: Mathematisches Institut, Universität Basel, Rheinsprung 21, CH-4051 Basel, Switzerland; e-mail: catherine.bandle@unibas.ch

J. v. Below: LMPA J. Liouville, EA 2597, Université du Littoral Côte d'Opale, 50, rue F. Buisson, B.P. 699, F-62228 Calais Cedex, France; e-mail: joachim.von.below@1mpa.univ-littoral.fr

W. Reichel: Mathematisches Institut, Universität Giessen, Arndtstrasse 2, D-35392 Giessen, Germany; e-mail: wolfgang.reichel@ math.uni-giessen.de

Mathematics Subject Classification (2000): 35J25, 35B50 
According to the classical theory there exists a unique solution for every $\lambda$ which does not coincide with an eigenvalue of

$$
-\Delta \varphi+q(x) \varphi=\lambda \varphi \quad \text { in } D, \quad \varphi_{n}=\sigma(x) \lambda \varphi \quad \text { on } \partial D .
$$

The first goal of this paper is to determine the range of $\lambda$-values for which positive $f$ and $g$ imply the positivity of the solution $u$. If such a property holds we say that (1.1) satisfies the positivity principle.

The positivity principle depends on the eigenvalue problem (1.2), which was analyzed in [4] for $\sigma \in C(\partial D)$ with $\sigma(x) \geq 0$. Later this was generalized in [2] to the case where $\sigma \in \mathbb{R}$ is an arbitrary real constant and finally in [3] to the case where $\sigma \in C(\partial D)$ has non-vanishing negative part. We briefly summarize the main results. For $v, w \in H^{1}(D)$ let

$$
\langle v, w\rangle=\int_{D}(\nabla v \cdot \nabla w+q(x) v w) d x, \quad a(v, w)=\int_{D} v w d x+\oint_{\partial D} \sigma(x) v w d s .
$$

There always exist infinitely many positive eigenvalues

$$
0<\lambda_{1}<\lambda_{2} \leq \cdots, \quad \lim _{n \rightarrow \infty} \lambda_{n}=\infty
$$

If $q(x) \geq 0$ and $\int_{D} q d x>0$ then $\langle\cdot, \cdot\rangle$ generates an equivalent norm on $H^{1}(D)$ and the lowest positive eigenvalue is characterized by the variational principle

$$
\lambda_{1}=\min \left\{\langle v, v\rangle: v \in H^{1}(D), a(v, v)=1\right\} .
$$

It is simple and the corresponding eigenfunction $\varphi_{1}$ is of constant sign in $\bar{D}$. Let

$$
\bar{\sigma}:=\frac{1}{|\partial D|} \int_{\partial D} \sigma(x) d s, \quad \sigma_{0}=-\frac{|D|}{|\partial D|} .
$$

If $q \equiv 0$ then $\lambda_{0}=0$ is an eigenvalue. If $\bar{\sigma}>\sigma_{0}$ then $\lambda_{0}=0$ plays the role of $\lambda_{1}$ (cf. Figure 1).

If $\sigma^{-}(x):=\max \{0,-\sigma(x)\} \not \equiv 0$ then there also exists a sequence of negative eigenvalues

$$
0>\lambda_{-1}>\lambda_{-2} \geq \cdots
$$

For space dimensions $N \geq 2, \lim _{n \rightarrow-\infty} \lambda_{n}=-\infty$, whereas in dimension $N=1$ there are at most two negative eigenvalues. In the case $q(x) \geq 0, \int_{D} q(x) d x>0$ the largest negative eigenvalue is given by

$$
\lambda_{-1}=-\min \left\{\langle v, v\rangle: v \in H^{1}(D), a(v, v)=-1\right\} .
$$

The eigenvalue $\lambda_{-1}$ is also simple, the corresponding eigenfunction $\varphi_{-1}$ has constant sign and does not vanish in $\bar{D}$. If $q \equiv 0$ and $\bar{\sigma}<\sigma_{0}$ then the eigenvalue $\lambda_{0}=0$ plays the role of $\lambda_{-1}$, whereas if $\bar{\sigma}>\sigma_{0}$ then it plays the role of $\lambda_{1}$ (cf. Figure 1).

Once the $\lambda$-region for which the positivity principle holds is understood, the question arises: what happens near the boundary of the positivity region? It turns out that there 
an anti-maximum principle holds, i.e. positive $f$ and $g$ imply that the solution of (1.1) is negative.

Our main results on the positivity and anti-maximum principle are stated and proved in Sections 2 and 3. Here we present them in the following table; see also Figure 1. First we have to distinguish between two cases: $\sigma(x) \geq 0$ and $\sigma^{-} \not \equiv 0$. Then the case $\sigma^{-} \not \equiv 0$ has to be further subdivided according to the potential $q$.

\begin{tabular}{|c|c|c|c|c|}
\hline & \multirow{2}{*}{$\sigma \geq 0$} & \multicolumn{3}{|c|}{$\sigma^{-} \not \equiv 0$} \\
\hline & & $\int_{D} q d x>0$ & & $q \equiv 0$ \\
\hline $\begin{array}{l}\text { positivity } \\
\text { principle }\end{array}$ & $\lambda<\lambda_{1}$ & $\lambda_{-1}<\lambda<\lambda_{1}$ & $\begin{array}{l}\bar{\sigma}<\sigma_{0}: \\
\bar{\sigma}>\sigma_{0}:\end{array}$ & $\begin{array}{c}0<\lambda<\lambda_{1} \\
\lambda_{-1}<\lambda<0\end{array}$ \\
\hline $\begin{array}{l}\text { anti-max } \\
\text { principle }\end{array}$ & $\lambda_{1}<\lambda<\lambda_{1}+\delta$ & $\begin{array}{c}\lambda_{-1}-\epsilon<\lambda<\lambda_{-1} \\
\lambda_{1}<\lambda<\lambda_{1}+\delta\end{array}$ & $\begin{array}{l}\bar{\sigma}<\sigma_{0} \\
\bar{\sigma}>\sigma_{0}\end{array}$ & $\begin{array}{c}-\epsilon<\lambda<0 \\
\lambda_{1}<\lambda<\lambda_{1}+\delta \\
\lambda_{-1}-\epsilon<\lambda<\lambda_{-1} \\
0<\lambda<\delta\end{array}$ \\
\hline
\end{tabular}

At the boundary $\lambda=\lambda_{ \pm 1}$ a solution to (1.1) for positive $f$ and $g$ can only exist if both vanish. In this case $u$ coincides with the eigenfunction $\varphi_{ \pm 1}$. Since both are of constant sign and can be taken either positive or negative it follows that neither the positivity nor the anti-maximum principle holds.

An interesting observation is that the positivity region is connected or disconnected according to $\int_{D} q d x>0$ or $q \equiv 0$ (cf. Figure 1 for the case where $\sigma \in \mathbb{R}$ does not depend on $x \in \partial D)$.
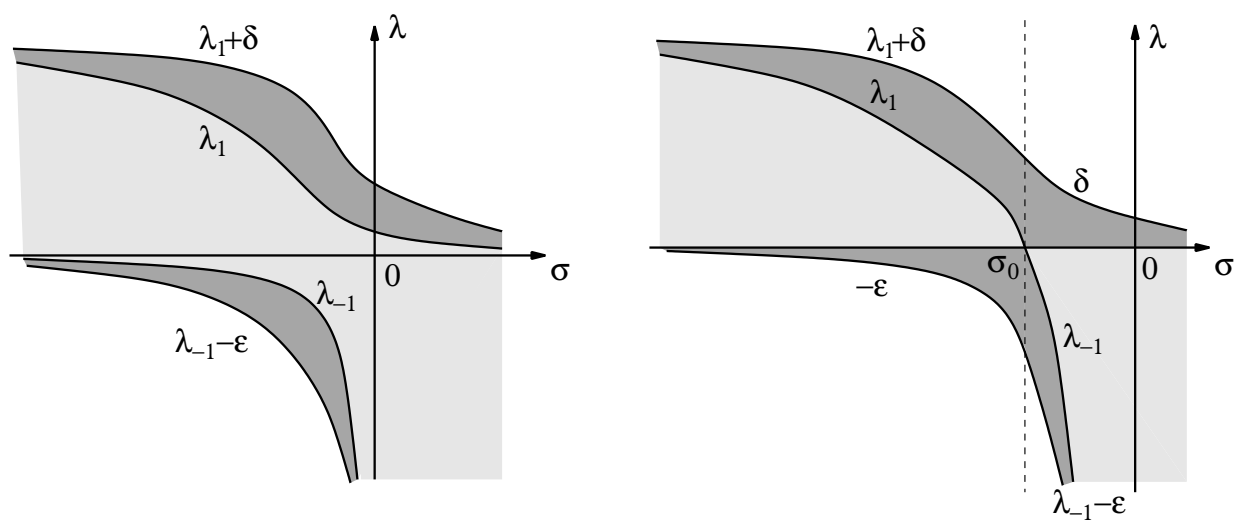

Fig. 1. Positivity, anti-max. principle. Left: $\int_{D} q d x>0$; right: $q \equiv 0$.

The anti-maximum principle was first studied by Clément and Peletier [5]. More recent studies on the anti-maximum principle are found in [1], [6]-[8], [10], [11], [14]. In 
[13] Hess and Kato studied the problem $-\Delta u=\lambda m(x) u$ in $D, u=0$ on $\partial D$ with a sign-changing coefficient $m(x)$, which corresponds to our coefficient $\sigma(x)$. They found a similar phenomenon of both positive and negative spectrum but the existence of the unbounded negative spectrum did not depend on the dimension $N$ of the space as in our case. Positivity and anti-maximum principles for Dirichlet problems $-\Delta u=\lambda m(x) u+f$ in $D, u=0$ on $\partial D$ with a sign-changing coefficient $m(x)$ are given in [10], [11] and [13].

It is known already from the work of Clément and Peletier [5] that in dimension $N=1$ one can expect the anti-maximum principle to be uniform in the sense that $\delta, \epsilon$ do not depend on $f$ and $g$. This is indeed the case, and moreover one can determine exactly the optimal $\lambda$-interval for the validity of the uniform anti-maximum principle. Such optimal anti-maximum principles are stated and proved in Section 4 . The boundaries of the optimal $\lambda$-intervals are determined through associated Dirichlet eigenvalues of (1.2), where one boundary value is changed from mixed to Dirichlet. Our results extend and complement those of [1], [10] and [14].

Finally, in Section 5 we apply the previous results to boundary value problems of the form

$$
-\Delta u+q(x) u=\alpha u+f \quad \text { in } D, \quad u_{n}=\beta u+g \quad \text { on } \partial D,
$$

where $\alpha$ and $\beta$ are real parameters. By means of our results on the positivity principle for (1.1) we determine the exact parameter region for which the positivity principle holds for (1.6.

In the Appendix we state and prove a Harnack-type inequality which is central for our results. For weak $H^{1}(D)$-solutions the Harnack-type inequality is the replacement for the strong maximum principle.

\section{Positivity principle}

Recall from [2], [3] that the eigenvalue problem (1.2] has a sequence of positive eigenvalues $\lambda_{k} \rightarrow \infty$ for $k \rightarrow \infty$. If $\sigma^{-} \not \equiv 0$ and if the space dimension is $N \geq 2$ then there also exists a sequence of negative eigenvalues with $\lambda_{k} \rightarrow-\infty$ as $k \rightarrow-\infty$ whereas in dimension $N=1$ there are at most two negative eigenvalues. Here we use the notation that $\lambda_{k}>(<,=) 0$ if $k>(<,=) 0$.

Our conditions for the positivity principle will be formulated such that the solutions of (1.1) are non-negative. Due to a strong maximum principle/Harnack-type inequality (see Appendix) this result can be strengthened in the following way: either $u \equiv 0$ or there exists $\delta=\delta(u)>0$ such that $u \geq \delta$ a.e. in $D$ and trace $u \geq \delta$ a.e. on $\partial D$.

In the statements of the following theorems we do not explicitly assume $\sigma^{-} \not \equiv 0$ because we want to include the case $\sigma(x) \geq 0$. Formally, this is achieved by setting $\lambda_{-1}=-\infty$ if $\sigma(x) \geq 0$. The positivity property in the case $\sigma(x) \geq 0$ may also be called the maximum principle, which we state next.

A function $u \in H^{1}(D)$ is called a weak supersolution of

$$
-\Delta u+Q(x) u \geq \lambda u \quad \text { in } D, \quad u_{n} \geq \Sigma(x) \lambda u \quad \text { on } \partial D
$$


provided

$\int_{D}(\nabla u \nabla v+Q(x) u v) d x \geq \int_{D} \lambda u v d x+\oint_{\partial D} \lambda \Sigma(x) u v d s \quad \forall v \in H^{1}(D)$ with $v \geq 0$.

If $\Sigma(x) \geq 0$ then the principle (first) eigenvalue $\Lambda_{\text {princ }}$ is given by

$\Lambda_{\text {princ }}=\min \left\{\int_{D}\left(|\nabla v|^{2}+Q(x) v^{2}\right) d x: v \in H^{1}(D), \int_{D} v^{2} d x+\oint_{\partial D} \Sigma(x) v^{2} d s=1\right\}$.

Note that $\Lambda_{\text {princ }}=0$ if $Q \equiv 0$, which is the reason why we call this eigenvalue $\Lambda_{\text {princ }}$ (and not $\Lambda_{1}$ ).

Lemma 1 (Maximum principle). Let $\Sigma(x) \geq 0$ and $0 \leq Q \in L^{\infty}(D)$. If $\lambda \in$ $\left(-\infty, \Lambda_{\text {princ }}\right)$ then every weak supersolution to 2.1 satisfies $u \geq 0$, and moreover, either $u \equiv 0$ or there exists $\delta=\delta(u)>0$ such that $u \geq \delta$ in $D$ and trace $u \geq \delta$ on $\partial D$.

The proof of $u \geq 0$ is standard and consists in using the test function $v=u^{-}$together with

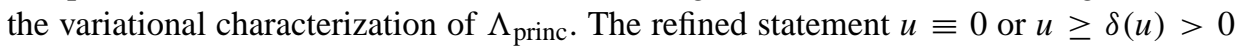
follows from Lemma 17(ii) in the Appendix. It might be interesting to note that the (almost) reverse conclusion also holds: if a weak supersolution to 2.1 satisfies $u \geq 0$ then necessarily $\lambda \in\left(-\infty, \Lambda_{\text {princ }}\right]$. The proof of this reverse statement is included in Theorem 2 below.

\subsection{The case $q(x) \geq 0, \int_{D} q d x>0$}

Recall the variational characterization 1.3 , 1.5 from the previous section. The case $\sigma(x) \geq 0$ is consistently covered since in this case the set of admissible functions in the definition of $\lambda_{-1}$ is empty and hence the infimum is $+\infty$.

Theorem 2. Let $0 \leq q \in L^{\infty}(D)$ with $\int_{D} q d x>0$ and assume $0 \leq f \in L^{2}(D)$ and $0 \leq g \in L^{2}(D)$.

(a) If $\lambda \in\left(\lambda_{-1}, \lambda_{1}\right)$ then the solution $u$ of 1.1 satisfies $u \geq 0$.

(b) If $u \geq 0$, $\neq \equiv$ is a supersolution of 1.1 then $\lambda \in\left[\lambda_{-1}, \lambda_{1}\right]$.

Proof. (a) The case $\sigma(x) \geq 0$ follows from the maximum principle of Lemma 1 . Therefore we assume $\sigma^{-} \not \equiv 0$ in the following. The case $\lambda=0$ is covered by the classical maximum principle for the Neumann problem. Hence we consider the two cases $\lambda \in\left(0, \lambda_{1}\right)$ and $\lambda \in\left(\lambda_{-1}, 0\right)$ separately.

Case 1: Let $\lambda \in\left(0, \lambda_{1}\right)$. Let $S=\max \left\{\|\sigma\|_{\infty}, 1\right\}$. Note that 1.1 is equivalent to

$$
\begin{aligned}
-\Delta u+(q(x)+(S-1) \lambda) u & =S \lambda u+f & & \text { in } D, \\
u_{n}+(S-\sigma(x)) \lambda u & =S \lambda u+g & & \text { on } \partial D .
\end{aligned}
$$

Let $K_{\lambda}$ be the operator given by

$$
K_{\lambda}: L^{2}(D) \times L^{2}(\partial D) \rightarrow H^{1}(D), \quad(h, k) \mapsto v,
$$


where $v$ is the unique solution of

$$
-\Delta v+(q(x)+(S-1) \lambda) v=h \quad \text { in } D, \quad v_{n}+(S-\sigma(x)) \lambda v=k \quad \text { on } \partial D .
$$

By a straightforward application of the maximum principle (cf. Lemma 1), the operator $K_{\lambda}$ is positive, and possesses a first eigenvalue $\alpha>0$ with a first eigenfunction $0<\varphi \in$ $H^{1}(D)$ which satisfies

$-\Delta \varphi+(q(x)+(S-1) \lambda) \varphi=\alpha \varphi \quad$ in $D, \quad \varphi_{n}+(S-\sigma(x)) \lambda \varphi=\alpha \varphi \quad$ on $\partial D$.

After testing (2.3) with $\varphi$ we obtain

$$
\int_{D}\left(|\nabla \varphi|^{2}+q(x) \varphi^{2}\right) d x=(\alpha+(1-S) \lambda) \int_{D} \varphi^{2} d x+\oint_{\partial D}(\alpha+(\sigma(x)-S) \lambda) \varphi^{2} d s .
$$

Let us show that $S \lambda<\alpha$. Assume for contradiction that $\alpha \leq S \lambda$. Then (2.4) implies

$$
\int_{D}\left(|\nabla \varphi|^{2}+q(x) \varphi^{2}\right) d x \leq \lambda\left(\int_{D} \varphi^{2} d x+\oint_{\partial D} \sigma(x) \varphi^{2} d x\right) .
$$

The variational characterization (1.3) of $\lambda_{1}$ implies $\lambda_{1} \leq \lambda$, which contradicts the hypothesis on $\lambda$. Hence we have proved that $S \lambda<\alpha$. Now we rewrite 2.2 as

$$
u=S \lambda K_{\lambda}(u, u)+K_{\lambda}(f, g) .
$$

If we introduce $\tilde{K}_{\lambda}: H^{1}(D) \rightarrow H^{1}(D)$ by $\tilde{K}_{\lambda} u=K_{\lambda}(u, u)$, then the previous equation is equivalent to

$$
\left(\mathrm{Id}-S \lambda \tilde{K}_{\lambda}\right) u=K_{\lambda}(f, g) .
$$

Since $0<S \lambda<\alpha$ the inverse of the operator Id $-S \lambda \tilde{K}_{\lambda}$ is given by the Neumann series $\sum_{k=0}^{\infty}\left(S \lambda \tilde{K}_{\lambda}\right)^{k}$ and is therefore a positive operator. This implies the claim of the theorem in Case 1.

Case 2: Let $\lambda \in\left(\lambda_{-1}, 0\right)$. Now we rewrite 1.1 as

$$
\begin{aligned}
-\Delta u+(q(x)-(S+1) \lambda) u & =-S \lambda u+f & & \text { in } D, \\
u_{n}-(S+\sigma(x)) \lambda u & =-S \lambda u+g & & \text { on } \partial D .
\end{aligned}
$$

Let $L_{\lambda}$ be the operator given by

$$
L_{\lambda}: L^{2}(D) \times L^{2}(\partial D) \rightarrow H^{1}(D), \quad(h, k) \mapsto v,
$$

where $v$ is the unique solution of

$$
-\Delta v+(q(x)-(S+1) \lambda) v=h \quad \text { in } D, \quad v_{n}-(S+\sigma(x)) \lambda v=k \quad \text { on } \partial D .
$$

Due to the maximum principle of Lemma 1 the operator $L_{\lambda}$ is positive with first eigenvalue $\beta>0$ and first eigenfunction $0<\psi \in H^{1}(D)$ satisfying

$-\Delta \psi+(q(x)-(S+1) \lambda) \psi=\beta \psi \quad$ in $D, \quad \psi_{n}-(S+\sigma(x)) \lambda \psi=\beta \psi \quad$ on $\partial D$. 
After testing 2.7) with $\psi$ and rearranging terms we obtain

$$
\left.\int_{D}\left(|\nabla \psi|^{2}+q(x) \psi^{2}\right) d x=\int_{D}(\beta+(S+1) \lambda)\right) \psi^{2} d x+\oint_{\partial D}(\beta+(S+\sigma(x)) \lambda) \psi^{2} d s .
$$

This implies that $S \lambda>-\beta$, since otherwise (2.8) leads to

$$
\int_{D}\left(|\nabla \psi|^{2}+q(x) \psi^{2}\right) d x \leq \lambda\left(\int_{D} \psi^{2} d x+\oint_{\partial D} \sigma(x) \psi^{2} d s\right) .
$$

The variational characterization (1.5) of $\lambda_{-1}$ implies $\lambda_{-1} \geq \lambda$, which contradicts the hypothesis on $\lambda$. Hence we have proved that $S \lambda>-\beta$. Note that (2.6) amounts to

$$
u=-S \lambda L_{\lambda}(u, u)+L_{\lambda}(f, g) .
$$

With the abbreviation $\tilde{L}_{\lambda}(u):=L_{\lambda}(u, u)$ the previous equation is equivalent to

$$
\left(\operatorname{Id}+S \lambda \tilde{L}_{\lambda}\right) u=L_{\lambda}(f, g) .
$$

Since $S \lambda>-\beta$ the inverse of the operator Id $+S \lambda \tilde{L}_{\lambda}$ is given by the Neumann series $\sum_{k=0}^{\infty}\left(-S \lambda \tilde{L}_{\lambda}\right)^{k}$ and thus it is positive. This finishes the proof of part (a) of the theorem.

(b) The following proof is inspired by Godoy et al. [10], where the idea is attributed to Hess [12]. Suppose (1.1) has a supersolution $u \geq 0, \not \equiv 0$. Since there exists $\delta>0$ such that $u \geq \delta$ in $D$ and trace $u \geq \delta$ on $\partial D$ we may write $u=e^{z}$ with a function $z \in H^{1}(D)$. For $v \in C^{\infty}(\bar{D})$ let us use $v^{2} e^{-z}$ as a test function for 1.1 . Thus we obtain

$$
\begin{aligned}
\int_{D}\left(-|v \nabla z-\nabla v|^{2}+\mid\right. & \left.\left.\nabla v\right|^{2}+q(x) v^{2}\right) d x \\
& \geq \int_{D}\left(\lambda v^{2}+f v^{2} e^{-z}\right) d x+\oint_{\partial D}\left(\sigma(x) \lambda v^{2}+g v^{2} e^{-z}\right) d s,
\end{aligned}
$$

which implies

$$
\int_{D}\left(|\nabla v|^{2}+q(x) v^{2}\right) d x \geq \lambda\left(\int_{D} v^{2} d x+\oint_{\partial D} \sigma(x) v^{2} d s\right) \quad \forall v \in C^{\infty}(\bar{D}) .
$$

The variational characterization of $\lambda_{-1}$ and $\lambda_{1}$ implies that necessarily $\lambda_{-1} \leq \lambda \leq \lambda_{1}$. This completes the proof of the theorem.

\subsection{The case $q(x) \equiv 0$}

Now we turn to the case $q \equiv 0$, where $\lambda_{0}=0$ is an eigenvalue. Therefore the variational characterization of the principal eigenvalues is different:

$$
\begin{aligned}
\lambda_{1} & =\min \left\{\int_{D}|\nabla v|^{2} d x: v \in H^{1}(D), a(v, 1)=0, a(v, v)=1\right\}, \\
\lambda_{-1} & =-\min \left\{\int_{D}|\nabla v|^{2} d x: v \in H^{1}(D), a(v, 1)=0, a(v, v)=-1\right\} .
\end{aligned}
$$

As before, $\sigma(x) \geq 0$ implies $\lambda_{-1}=-\infty$. 
The positivity principle of this section relies on the following result, which was proved in [3]. Recall the definition (1.4) of $\bar{\sigma}, \sigma_{0}$ from the introduction.

Proposition 3. If $\bar{\sigma} \in\left(-\infty, \sigma_{0}\right)$ then the eigenvalue $\lambda_{1}$ is simple and the eigenfunction corresponding to $\lambda_{1}$ has constant sign. If $\sigma^{-} \not \equiv 0$ and $\bar{\sigma} \in\left(\sigma_{0}, \infty\right)$ then $\lambda_{-1}$ is simple and the eigenfunction corresponding to $\lambda_{-1}$ has constant sign.

Theorem 4. Let $q \equiv 0$ and assume $0 \leq f \in L^{2}(D), 0 \leq g \in L^{2}(D)$.

(i) $\bar{\sigma} \in\left(-\infty, \sigma_{0}\right)$ :

(a) If $\lambda \in\left(0, \lambda_{1}\right)$ then the solution $u$ of (1.1) satisfies $u \geq 0$.

(b) If $u \geq 0, \not \equiv 0$ is a supersolution of (1.1) then $\lambda \in\left[0, \lambda_{1}\right]$.

(ii) $\bar{\sigma} \in\left(\sigma_{0}, \infty\right)$ :

(a) If $\lambda \in\left(\lambda_{-1}, 0\right)$ then the solution $u$ of (1.1) satisfies $u \geq 0$.

(b) If $u \geq 0, \not \equiv 0$ is a supersolution of (1.1) then $\lambda \in\left[\lambda_{-1}, 0\right]$.

(iii) $\bar{\sigma}=\sigma_{0}$ :

(a) There is no value of $\lambda$ such that (1.1) has the positivity property.

(b) If $u \geq 0, \not \equiv 0$ is a supersolution of (1.1) then $\lambda=0$.

Proof. The case $\sigma(x) \geq 0$ falls within case (ii) and is covered by the maximum principle of Lemma 1. Hence we may assume $\sigma^{-} \not \equiv 0$.

Case (i), part (a): Since the proof is very similar to Case 1 in Theorem 2 let us indicate the differences. One rewrites (1.1) as 2.2 and introduces the same positive operator $K_{\lambda}$ with the first eigenvalue $\alpha$ satisfying 2.3]. One needs to show that $S \lambda<\alpha$. This is where a different argument is needed. Assuming for contradiction as before that $\alpha \leq S \lambda$ we obtain 2.5. However, $\varphi$ does not satisfy $a(\varphi, 1)=0$ and hence cannot be inserted into the variational characterization of $\lambda_{1}$. Instead, we define

$$
\tilde{\varphi}=\varphi-P \varphi, \quad P \varphi=\frac{\int_{D} \varphi d x+\oint_{\partial D} \sigma(x) \varphi d s}{|D|+\bar{\sigma}|\partial D|} .
$$

Clearly $a(\tilde{\varphi}, 1)=0$. Rewriting 2.5 we obtain

$$
\begin{aligned}
\int_{D}|\nabla \tilde{\varphi}|^{2} d x \leq & \lambda\left(\int_{D} \tilde{\varphi}^{2} d x+\oint_{\partial D} \sigma(x) \tilde{\varphi}^{2} d x\right) \\
& +\lambda(P \varphi)^{2}(|D|+\bar{\sigma}|\partial D|)+2 \lambda P \varphi(\underbrace{\int_{D} \tilde{\varphi} d x+\oint_{\partial D} \sigma(x) \tilde{\varphi} d s}_{=0}),
\end{aligned}
$$

and since $\bar{\sigma}<\sigma_{0}$ this implies by the variational characterization of $\lambda_{1}$ the contradiction $\lambda_{1} \leq \lambda$. The proof continues exactly as in Case 1 of Theorem 2 .

Case (ii), part (a): The proof resembles the one of Case 2 in Theorem 2 using the operator $L_{\lambda}$. One only needs to prove $S \lambda>-\beta$. Assume the contrary. With the help of the projection $\tilde{\psi}=\psi-P \psi$ one can rewrite 2.9 as above, use the variational characterization of $\lambda_{-1}$ and get a contradiction. The proof is then completed as in Case 2 of Theorem 2 . 
Cases ( $i)$ and (ii), part (b): As in the proof of Theorem 2 the existence of a non-negative solution $u$ of (1.1) leads to

$$
\int_{D}|\nabla v|^{2} d x \geq \lambda\left(\int_{D} v^{2} d x+\oint_{\partial D} \sigma(x) v^{2} d s\right) \quad \forall v \in C^{\infty}(\bar{D}),
$$

in particular for those $v$ with $a(v, 1)=0$. This implies that

$$
\lambda_{-1} \leq \lambda \leq \lambda_{1}
$$

However, more precise information on the location of $\lambda$ is needed. Note that in the case $\bar{\sigma}<\sigma_{0}$ one has

$$
0=\lambda_{0}=\min \left\{\int_{D}|\nabla v|^{2} d x: a(v, v)=-1\right\}
$$

with $v=$ const as a minimizer. Hence 2.11) implies that besides 2.12) also $\lambda \geq 0$ has to hold. In the case $\sigma_{0}<\bar{\sigma}$ notice that

$$
0=\lambda_{0}=\min \left\{\int_{D}|\nabla v|^{2} d x: a(v, v)=1\right\} .
$$

Thus together with 2.12) also $\lambda \leq 0$ has to hold.

Case (iii): Part (a) follows once part (b) is shown, since then the only value of $\lambda$ for which the positivity property could hold is $\lambda=0$. But even for $\lambda=0$ the positivity property cannot hold as we may subtract arbitrary constants from solutions. So it remains to show part (b): as before we obtain inequality 2.11). We will show that in this case the following two characterizations of $\lambda_{0}=0$ hold simultaneously:

$$
\begin{aligned}
0 & =\inf \left\{\int_{D}|\nabla v|^{2} d x: a(v, v)=-1\right\} \\
& =\inf \left\{\int_{D}|\nabla v|^{2} d x: a(v, v)=1\right\},
\end{aligned}
$$

where neither of the two minimization problems has a minimizer. Together with 2.11) this implies that necessarily $\lambda=0$. So let us show (2.13) and 2.14). Let $w$ be a solution of

$$
-\Delta w=1 \quad \text { in } D, \quad w_{n}=\sigma(x) \text { on } \partial \Omega,
$$

which exists only in the case $\bar{\sigma}=\sigma_{0}$. Next define $v_{t}=1+t w$ for $t \in \mathbb{R}$. Then $\int_{D}\left|\nabla v_{t}\right|^{2} d x=\int_{D} t^{2}|\nabla w|^{2} d x$ and

$$
a\left(v_{t}, v_{t}\right)=a(1,1)+2 t a(w, 1)+t^{2} a(w, w)=2 t \int_{D}|\nabla w|^{2} d x+t^{2} a(w, w) .
$$

Let $\tilde{v}_{t}=v_{t} / \sqrt{\left|a\left(v_{t}, v_{t}\right)\right|}$. Then

$$
\lim _{t \rightarrow 0} \int_{D}\left|\nabla \tilde{v}_{t}\right|^{2} d x=\lim _{t \rightarrow 0} \frac{\int_{D}\left|\nabla v_{t}\right|^{2} d x}{\left|a\left(v_{t}, v_{t}\right)\right|}=0
$$

and $a\left(\tilde{v}_{t}, \tilde{v}_{t}\right)=+1$ or -1 if $t>0$ or $t<0$. Hence if $t \rightarrow 0$ then $\tilde{v}_{t}$ is a minimizing family for 2.13 if $t>0$ and for 2.14$)$ if $t<0$. This finishes the proof of the claim. 


\section{Anti-maximum principles}

In this section we consider (1.1) with $f, g \geq 0$ and $\lambda$ lying outside the region where the positivity principle holds. One expects by the results of [5] a so called "anti-maximum principle": if $q \geq 0, \int_{D} q d x>0$ and $\lambda$ is a little larger than $\lambda_{1}$ or a little smaller than $\lambda_{-1}$ then the solution of $(1.1)$ is negative. The situation for $q \equiv 0$ is again more complicated. As before we treat the case $\sigma(x) \geq 0$ by setting $\lambda_{-1}=-\infty$.

\subsection{The case $q(x) \geq 0, \int_{D} q d x>0$}

Theorem 5. Let $0 \leq q \in L^{\infty}(D)$ with $\int_{D} q d x>0$. Suppose that $0 \leq f \in L^{p_{1}}(D)$ with $p_{1}>N / 2, p_{1} \geq 2$ and $0 \leq g \in L^{p_{2}}(\partial D)$ with $p_{2}>N-1, p_{2} \geq 2$, and assume additionally that $f \not \equiv 0$ or $g \not \equiv 0$. Then there exists $\delta=\delta(f, g, \sigma)>0, \epsilon=$ $\epsilon(f, g, \sigma)>0$ such that if $\lambda \in\left(\lambda_{-1}-\epsilon, \lambda_{-1}\right) \cup\left(\lambda_{1}, \lambda_{1}+\delta\right)$ then the solution $u$ of 1.1] satisfies $u<0$ in $\bar{D}$.

Proof. Case 1: Let $\lambda_{1}<\lambda$ and assume moreover that $\lambda<\lambda_{2}-\gamma$ for some fixed small $\gamma>0$. Then 1.1 has a unique solution $u \in H^{1}(D)$. Recall from the Hilbert space theory of [2], [3] that $H^{1}(D)=\operatorname{span}\left[\varphi_{1}\right] \oplus V$, where $\operatorname{span}\left[\varphi_{1}\right]$ and $V$ are orthogonal both with respect to the bilinear form $a(\cdot, \cdot)$ and the inner product $\langle\cdot, \cdot\rangle$. We assume the normalization $a\left(\varphi_{1}, \varphi_{1}\right)=1$. From [2], [3] we also know that $\varphi_{1}$ has constant sign and that there is a $\kappa>0$ such that $\varphi_{1} \geq \kappa$ in $D$. By using the splitting of the space the solution $u$ of $(1.1)$ is decomposed as $u=\alpha \varphi_{1}+v$. A direct computation yields

$$
\alpha=\frac{\int_{D} f \varphi_{1} d x+\oint_{\partial D} g \varphi_{1} d s}{\lambda_{1}-\lambda}
$$

and

$$
-\Delta v+q(x) v=\lambda v+f^{\vdash} \quad \text { in } D, \quad v_{n}=\sigma(x) \lambda v+g^{\vdash} \quad \text { on } \partial D,
$$

where 1

$$
\begin{aligned}
& f^{\vdash}:=f-\left(\int_{D} f \varphi_{1} d x+\oint_{\partial D} g \varphi_{1} d s\right) \varphi_{1}, \\
& g^{\vdash}:=g-\sigma(x)\left(\int_{D} f \varphi_{1} d x+\oint_{\partial D} g \varphi_{1} d s\right) \varphi_{1} .
\end{aligned}
$$

Note that $f^{\vdash}, g^{\vdash}$ lie in the same $L^{p}$-spaces as $f, g$ since $\varphi_{1} \in L^{\infty}(D)$ and trace $\varphi_{1} \in$ $L^{\infty}(\partial D)$. Let us introduce the compact operator $K: L^{2}(D) \times L^{2}(\partial D) \rightarrow H^{1}(D)$ defined by $K(h, k)=z$ with $-\Delta z+q(x) z=h$ in $D$ and $z_{n}=k$ on $\partial D$. One finds easily that $K\left(f^{\vdash}, g^{\vdash}\right) \in V=\operatorname{span}\left[\varphi_{1}\right]^{\perp}$. Moreover the operator $\tilde{K} v=K(v, \sigma v)$ mapping $V \rightarrow V$ is well-defined. Therefore (3.1) amounts to

$$
(\operatorname{Id}-\lambda \tilde{K}) v=K\left(f^{\vdash}, g^{\vdash}\right)
$$

1 The definition of $f^{\vdash}, g^{\vdash}$ implies that $b\left(f^{\vdash}, g^{\vdash}, \varphi_{1}\right)=0$ with $b(f, g, v):=\int_{D} f v d x+$ $\oint_{\partial D} g v d s$ (see also the proof of Theorem 6 . 
and the solution $v$ of 3.2 can be found by inverting $\operatorname{Id}-\lambda \tilde{K}$ on the space $V$. Since the values of $\lambda$ satisfy $\lambda \in\left(\lambda_{1}, \lambda_{2}-\gamma\right)$ there exists a constant $C$ independent of $\lambda$ such that

$$
\|v\|_{H^{1}(D)} \leq C\left(\|f\|_{L^{2}(D)}+\|g\|_{L^{2}(\partial D)}\right) .
$$

Lemma 17 in the Appendix applied to (3.1) implies that

$$
\|v\|_{L^{\infty}(D)} \leq \bar{C}\left(\|v\|_{L^{2}(D)}+\|f\|_{L^{p_{1}(D)}}+\|g\|_{L^{p_{2}(\partial D)}}\right)
$$

uniformly in $\lambda \in\left(\lambda_{1}, \lambda_{2}-\gamma\right)$. With $\tilde{p}_{1}=\max \left\{2, p_{1}\right\}, \tilde{p}_{2}=\max \left\{2, p_{2}\right\}$ we can combine the two estimates into

$$
\|v\|_{L^{\infty}(D)} \leq \bar{C}\left(\|f\|_{L^{\tilde{p}_{1}(D)}}+\|g\|_{L^{\tilde{p}_{2}(\partial D)}}\right) .
$$

With the help of the decomposition $u=\alpha \varphi_{1}+v$ and the estimate $\varphi_{1} \geq \kappa$ we obtain

$$
u \leq \kappa \frac{\int_{D} f \varphi_{1} d x+\oint_{\partial D} g \varphi_{1} d s}{\lambda_{1}-\lambda}+\bar{C}\left(\|f\|_{L^{\tilde{p}_{1}(D)}}+\|g\|_{L^{\tilde{p}_{2}(\partial D)}}\right) \quad \text { in } D,
$$

which can be made uniformly negative in $D$ provided $\lambda \in\left(\lambda_{1}, \lambda_{1}+\delta(f, g)\right)$ with a positive but sufficiently small value of $\delta(f, g)$.

Case 2: Let $\lambda<\lambda_{-1}$ and assume further that $\lambda>\lambda_{-2}+\gamma$ for some fixed small $\gamma>0$. The unique solution $u \in H^{1}(D)$ of 1.1 has the orthogonal decomposition $u=\alpha \varphi_{-1}+v$. If we use the normalization $a\left(\varphi_{-1}, \varphi_{-1}\right)=-1$ then $\alpha$ is given by

$$
\alpha=\frac{\int_{D} f \varphi_{-1} d x+\oint_{\partial D} g \varphi_{-1} d s}{\lambda-\lambda_{-1}} .
$$

The function $\varphi_{-1}$ has constant sign and is bounded below by a positive constant $\kappa>0$. As in Case 1, one shows that $v$ is bounded in $L^{\infty}(D)$ uniformly for $\lambda \in\left(\lambda_{-2}+\gamma, \lambda_{-1}\right)$. Hence, if $\lambda$ is sufficiently close to $\lambda_{-1}$ the function $\alpha \varphi_{-1}$ in the decomposition of $u$ is sufficiently negative to make $u$ uniformly negative in $D$.

\subsection{The case $q(x) \equiv 0$}

Theorem 6. Let $q \equiv 0$ and define $\bar{\sigma}=|\partial D|^{-1} \int_{\partial D} \sigma(x) d s$. Suppose that $0 \leq f \in$ $L^{p_{1}}(D)$ with $p_{1}>N / 2, p_{1} \geq 2$ and $0 \leq g \in L^{p_{2}}(D)$ with $p_{2}>N-1, p_{2} \geq 2$, and assume additionally that $f \not \equiv 0$ or $g \not \equiv 0$. Then there exists $\delta=\delta(f, g, \sigma)>0$ and $\epsilon=\epsilon(f, g, \sigma)>0$ such that the solution $u$ of 1.1 satisfies $u<0$ in $\bar{D}$ provided

(i) $\bar{\sigma} \in\left(-\infty, \sigma_{0}\right)$ and $\lambda \in(-\epsilon, 0) \cup\left(\lambda_{1}, \lambda_{1}+\delta\right)$,

(ii) $\bar{\sigma} \in\left(\sigma_{0}, \infty\right)$ and $\lambda \in\left(\lambda_{-1}-\epsilon, \lambda_{-1}\right) \cup(0, \delta)$,

(iii) $\bar{\sigma}=\sigma_{0}$ and $\lambda \in(-\epsilon, 0) \cup(0, \delta)$.

Proof. Cases (i) and (ii): The proofs are similar to the proof of Theorem 5. We illustrate only case (i). For $\bar{\sigma}<\sigma_{0}$ we know from [2], [3] that $\lambda_{1}$ is simple with an eigenfunction 
$\varphi_{1} \geq \kappa>0$ in $D$. Assume the normalization $a\left(\varphi_{1}, \varphi_{1}\right)=1$. We use the splitting

$$
H^{1}(D)=\operatorname{span}\left[\varphi_{1}\right] \oplus \operatorname{span}[1] \oplus V
$$

into three orthogonal parts, i.e. the unique solution $u \in H^{1}(D)$ of $(1.1)$ is decomposed into $u=\alpha \varphi_{1}+\beta+v$. The values of $\alpha$ and $\beta$ are given by

$$
\alpha=\frac{\int_{D} f \varphi_{1} d x+\oint_{\partial D} g \varphi_{1} d s}{\lambda_{1}-\lambda}, \quad \beta=-\frac{\int_{D} f d x+\oint_{\partial D} g d s}{\lambda\left(|D|+\int_{\partial D} \sigma(x) d s\right)}
$$

and $v$ solves

$$
-\Delta v=\lambda v+\lambda \beta+f^{\vdash} \quad \text { in } D, \quad v_{n}=\sigma(x) \lambda v+\sigma(x) \lambda \beta+g^{\vdash} \quad \text { on } \partial D
$$

with $f^{\vdash}, g^{\vdash}$ as in the proof of Theorem 5 On the space $\mathcal{W}=\left\{(h, k) \in L^{2}(D) \times L^{2}(\partial D)\right.$ : $\left.\int_{D} h d x+\oint_{\partial D} k d s=0=\int_{D} h \varphi_{1} d x+\oint_{\partial D} k \varphi_{1} d s\right\}$ we define the operator $K: \mathcal{W} \rightarrow V$ by $K(h, k)=z$ with $-\Delta z=h$ in $D, z_{n}=k$ on $\partial D$. Moreover $\tilde{K}: V \rightarrow V$ is defined by $\tilde{K} v=K(v, \sigma v)$. If we note (by a standard computation) that $\left(\lambda \beta+f^{\vdash}, \sigma \lambda \beta+g^{\vdash}\right) \in \mathcal{W}$ then (3.4) is equivalent to

$$
(\mathrm{Id}-\lambda \tilde{K}) v=K\left(\lambda \beta+f^{\vdash}, \sigma \lambda \beta+g^{\vdash}\right) .
$$

As long as $\lambda$ is bounded away from $\lambda_{-1}$ and $\lambda_{2}$ we get the estimates

$$
\|v\|_{H^{1}(D)} \leq C\left(\|f\|_{L^{2}(D)}+\|g\|_{L^{2}(\partial D)}\right)
$$

and

$$
\|v\|_{L^{\infty}(D)} \leq \bar{C}\left(\|u\|_{L^{2}(D)}+\|f\|_{L^{p_{1}(D)}}+\|g\|_{L^{p_{2}(\partial D)}}\right)
$$

uniformly for $\lambda \in\left[\lambda_{-1}+\gamma, \lambda_{2}-\gamma\right]$. Recalling that $|D|+\int_{\partial D} \sigma(x) d s<0$ if $\bar{\sigma}<\sigma_{0}$ we see from (3.3) that $u$ will be negative if either $\lambda$ is in a small right neighborhood of $\lambda_{1}$ or if $\lambda$ is in a small left neighborhood of 0 .

Case (iii): In this case (cf. [2], [3]) the space $H^{1}(D)$ has the decomposition

$$
H^{1}(D)=\operatorname{span}[1] \oplus \operatorname{span}[w] \oplus \mathcal{V}_{w},
$$

where $w$ solves $-\Delta w=1$ in $D, w_{n}=\sigma(x)$ on $\partial D$ and $\mathcal{V}_{w}=\left\{v \in H^{1}(D): a(v, 1)=\right.$ $a(v, w)=0\}$. Note however that $\operatorname{span}[1]$ and $\operatorname{span}[w]$ are not orthogonal. To facilitate notation let

$$
b: L^{2}(D) \times L^{2}(\partial D) \times H^{1}(D) \rightarrow \mathbb{R}, \quad(f, g, v) \mapsto \int_{D} f v d x+\oint_{\partial D} g v d s .
$$

The solution of (1.1) can accordingly be split into three parts, i.e., $u=\alpha+\beta w+v$, where

$$
\alpha=-\frac{b(f, g, 1)}{\lambda^{2} a(w, 1)}-\frac{b(f, g, w)}{\lambda a(w, 1)}+\frac{b(f, g, 1) a(w, w)}{\lambda a(w, 1)^{2}}, \quad \beta=-\frac{b(f, g, 1)}{\lambda a(w, 1)} .
$$


Note that $a(w, 1)=\int_{D}|\nabla w|^{2} d x>0$. The remaining equation for $v$ is

$$
\begin{aligned}
-\Delta v & =\frac{b(f, g, w)}{a(w, 1)}+\frac{b(f, g, 1) a(w, w)}{a(w, 1)^{2}}+\lambda v+f-\frac{b(f, g, 1)}{a(w, 1)} w \quad \text { in } D \\
v_{n} & =-\sigma \frac{b(f, g, w)}{a(w, 1)}+\sigma \frac{b(f, g, 1) a(w, w)}{a(w, 1)^{2}}+\sigma \lambda v+g-\sigma \frac{b(f, g, 1)}{a(w, 1)} w \quad \text { on } \partial D .
\end{aligned}
$$

Define the space $\mathcal{W}_{w}=\left\{(h, k) \in L^{2}(D) \times L^{2}(\partial D): \int_{D} h d x+\oint_{\partial D} k d s=0=\right.$ $\left.\int_{D} h w d x+\oint_{\partial D} k w d s\right\}$. On $\mathcal{W}_{w}$ let the operator $K: \mathcal{W}_{w} \rightarrow \mathcal{V}_{w}$ be given by $K(h, k):=z$, where $z \in \mathcal{V}_{w}$ is the unique solution of $-\Delta z=h$ in $D, z_{n}=k$ on $\partial D$ (cf. [2], [3]). Likewise, let $\tilde{K}: \mathcal{V}_{w} \rightarrow \mathcal{V}_{w}$ be defined by $\tilde{K} v=K(v, \sigma v)$. Thus 3.5 -3.6 is equivalent to

$$
\begin{aligned}
(\operatorname{Id}-\lambda \tilde{K}) v=K( & -\frac{b(f, g, w)}{a(w, 1)}+\frac{b(f, g, 1) a(w, w)}{a(w, 1)^{2}}+f-\frac{b(f, g, 1)}{a(w, 1)} w, \\
& \left.-\sigma \frac{b(f, g, w)}{a(w, 1)}+\sigma \frac{b(f, g, 1) a(w, w)}{a(w, 1)^{2}}+g-\sigma \frac{b(f, g, 1)}{a(w, 1)} w\right),
\end{aligned}
$$

if one verifies by a standard computation that the argument of $K$ on the right-hand side belongs to $\mathcal{W}_{w}$. Now the $L^{2}$ and $L^{\infty}$-bounds on $v$ follow as before provided $\lambda$ is bounded away from $\lambda_{-1}$ and $\lambda_{1}$. Likewise $\|\beta w\|_{\infty} \leq \mathrm{const}\left|\lambda^{-1}\right|\left(\|f\|_{L^{2}(D)}+\|g\|_{L^{2}(\partial D)}\right)$. Thus, negativity of $u$ is a consequence of the $1 / \lambda^{2}$-term in $\alpha$ provided $\lambda$ is sufficiently small but non-zero.

\section{Uniform anti-maximum principles}

If the dimension $N$ is 1 and $D=(0, L)$ then $(1.1)$ becomes

$$
\begin{gathered}
-u^{\prime \prime}+q(x) u=\lambda u+f \quad \text { in }(0, L), \\
-u^{\prime}(0)=\sigma_{1} \lambda u(0)+g_{1}, \quad u^{\prime}(L)=\sigma_{2} \lambda u(L)+g_{2} .
\end{gathered}
$$

It is known already from the work of Clément and Peletier [5] that in dimension $N=1$ one can expect the anti-maximum principle to be uniform in the sense that $\delta, \epsilon$ in Theorems 5 and 6 do not depend on $f$ and $g$. This is indeed the case, and moreover one can determine exactly the optimal $\lambda$-interval for the validity of the uniform anti-maximum principle.

Previously, such optimal $\lambda$-intervals were determined variationally by Arias et al. [1] and Godoy et al. [10] through the values $\bar{\lambda}, \underline{\lambda}$ (cf. Lemma 8). Another approach was given by Reichel [14] through the associated eigenvalue problems $\left(D_{L}\right),\left(D_{0}\right)$ below. Thanks to new observations we can now bring together these two approaches (cf. Lemma 9), and thus get explicit formulas for the optimal $\lambda$-interval. 
To formulate our results we need the following associated boundary value problems introduced in [14]. Note that one boundary value is changed from mixed to Dirichlet.

$$
\left(D_{L}\right)\left\{\begin{array} { l } 
{ - u ^ { \prime \prime } + q ( x ) u = \lambda u \quad \text { in } ( 0 , L ) , } \\
{ - u ^ { \prime } ( 0 ) = \sigma _ { 1 } \lambda u ( 0 ) , } \\
{ u ( L ) = 0 , }
\end{array} \quad ( D _ { 0 } ) \left\{\begin{array}{l}
-u^{\prime \prime}+q(x) u=\lambda u \quad \text { in }(0, L), \\
u(0)=0, \\
u^{\prime}(L)=\sigma_{2} \lambda u(L) .
\end{array}\right.\right.
$$

Both problems have a sequence of positive eigenvalues $\lambda_{k}^{L}, \lambda_{k}^{0}$ tending to $+\infty$ as $k \rightarrow \infty$. Negative eigenvalues may not always exist. This is explained at the beginning of the following two sections.

4.1. The case $q(x) \geq 0, \int_{0}^{L} q d x>0$

We recall from Bandle and Reichel [3] that negative eigenvalues exist:

\begin{tabular}{|c|c|c|c|}
\hline \multirow{2}{*}{ Conditions on $\sigma$} & \multicolumn{3}{|c|}{ Negative eigenvalues } \\
\cline { 2 - 4 } & for $4.1-4.2$ & for $\left(D_{L}\right)$ & for $\left(D_{0}\right)$ \\
\hline$\sigma_{1}, \sigma_{2}<0$ & $\lambda_{-2}<\lambda_{-1}$ & $\lambda_{-1}^{L}$ & $\lambda_{-1}^{0}$ \\
\hline$\sigma_{1}<0 \leq \sigma_{2}$ & $\lambda_{-1}$ & $\lambda_{-1}^{L}$ & no neg. ev \\
\hline $0 \leq \sigma_{1}, \sigma_{2}$ & no neg. ev & no neg. ev & no neg. ev \\
\hline
\end{tabular}

We define the missing negative eigenvalues as $-\infty$. For simplicity we do not consider the case $\sigma_{2}<0 \leq \sigma_{1}$ since it is essentially the same as $\sigma_{1}<0 \leq \sigma_{2}$.

Theorem 7. Let $0 \leq q \in L^{\infty}(0, L)$ with $\int_{0}^{L} q d x>0$ and let

$$
\lambda \in\left[\max \left\{\lambda_{-1}^{L}, \lambda_{-1}^{0}\right\}, \lambda_{-1}\right) \cup\left(\lambda_{1}, \min \left\{\lambda_{1}^{L}, \lambda_{1}^{0}\right\}\right] .
$$

Suppose that $0 \leq f \in L^{1}(0, L)$ and $g_{1}, g_{2} \geq 0$ and assume additionally $f \neq 0$ or $g_{1}, g_{2}>0$. Then the solution $u$ of (4.1) -4.2 satisfies $u<0$ in $[0, L]$. Moreover, the above $\lambda$-interval is optimal for the uniform anti-maximum principle.

The proof will be done with the help of the following two lemmas.

Lemma 8. Let $0 \leq q \in L^{\infty}(0, L)$ with $\int_{0}^{L} q d x>0$ and define

$$
\begin{aligned}
& \bar{\lambda}=\inf \left\{\int_{0}^{L}\left(v^{\prime 2}+q(x) v^{2}\right) d x: v \in H^{1}(0, L) \text { has a zero and } a(v, v)=1\right\}, \\
& \underline{\lambda}=-\inf \left\{\int_{0}^{L}\left(v^{\prime 2}+q(x) v^{2}\right) d x: v \in H^{1}(0, L) \text { has a zero and } a(v, v)=-1\right\},
\end{aligned}
$$

where $a(v, w)=\int_{0}^{L} v w d x+\sigma_{1} v(0) w(0)+\sigma_{2} v(L) w(L)$. Then $\bar{\lambda}$ is attained and $\lambda_{1}<$ $\bar{\lambda}<\lambda_{2}$. If either $\sigma_{1}$ or $\sigma_{2}$ is negative then $\underline{\lambda}$ is attained and $\lambda_{-2}<\underline{\lambda}<\lambda_{-1}$. The extremal functions for both extremal values have exactly one zero in $[0, L]$. 
Proof. The value $\bar{\lambda}$ is always finite. The value $\underline{\lambda}$ is finite if at least one of $\sigma_{1}, \sigma_{2}$ is negative. Otherwise $\underline{\lambda}=-\infty$. Provided the extremal values $\bar{\lambda}, \underline{\lambda}$ are finite the existence of extremal functions is standard since $H^{1}(0, L)$ embeds compactly into $C([0, L])$. Let $\bar{u}, \underline{u}$ be such extremal functions. Then $\bar{u}\left(x_{0}\right)=\underline{u}\left(y_{0}\right)=0$ for some $x_{0}, y_{0} \in[0, L]$. For a given point $z_{0} \in[0, L]$ define the space $V_{z_{0}}=\left\{v \in H^{1}(0, L): v\left(z_{0}\right)=0\right\}$, i.e., $\bar{u} \in V_{x_{0}}$ and $\underline{u} \in V_{y_{0}}$. Moreover, $\bar{u}, \underline{u}$ are extremal functions for

$$
\begin{aligned}
& \bar{\lambda}^{*}=\inf \left\{\int_{0}^{L}\left(v^{\prime 2}+q(x) v^{2}\right) d x: v \in V_{x_{0}} \text { and } a(v, v)=1\right\}, \\
& \underline{\lambda}^{*}=-\inf \left\{\int_{0}^{L}\left(v^{\prime 2}+q(x) v^{2}\right) d x: v \in V_{y_{0}} \text { and } a(v, v)=-1\right\} .
\end{aligned}
$$

Clearly $\bar{\lambda}=\bar{\lambda}^{*}, \underline{\lambda}=\underline{\lambda}^{*}$. Hence the following Euler equations hold:

$$
\langle\bar{u}, \phi\rangle=\bar{\lambda} a(\bar{u}, \phi) \quad \text { for all } \phi \in V_{x_{0}}, \quad\langle\underline{u}, \psi\rangle=\underline{\lambda} a(\underline{u}, \psi) \quad \text { for all } \psi \in V_{y_{0}},
$$

and standard regularity implies that $\bar{u}$ satisfies

$$
\begin{gathered}
-u^{\prime \prime}+q(x) u=\bar{\lambda} u \quad \text { in }\left(0, x_{0}\right) \cup\left(x_{0}, L\right), \\
-u^{\prime}(0)=\sigma_{1} \bar{\lambda} u(0), \quad u\left(x_{0}\right)=0, \quad u^{\prime}(L)=\sigma_{2} \bar{\lambda} u(L),
\end{gathered}
$$

and $\underline{u}$ satisfies

$$
\begin{gathered}
-u^{\prime \prime}+q(x) u=\underline{\lambda} u \quad \text { in }\left(0, y_{0}\right) \cup\left(y_{0}, L\right), \\
-u^{\prime}(0)=\sigma_{1} \underline{\lambda} u(0), \quad u\left(y_{0}\right)=0, \quad u^{\prime}(L)=\sigma_{2} \underline{\lambda} u(L) .
\end{gathered}
$$

Note that in the case $x_{0} \in\{0, L\}$ or $y_{0} \in\{0, L\}$ the Dirichlet boundary condition replaces the mixed boundary condition. Let us show that $\bar{u}$ has exactly one zero. The proof for $\underline{u}$ is the same. So assume $\bar{u} \in V_{x_{0}} \cap V_{x_{1}}$ for $x_{0}, x_{1} \in[0, L]$ with $x_{0} \neq x_{1}$. Then

$$
\langle\bar{u}, \phi\rangle=\bar{\lambda} a(\bar{u}, \phi) \quad \text { for all } \phi \in V_{x_{0}} \oplus V_{x_{1}} .
$$

But $V_{x_{0}} \oplus V_{x_{1}}=H^{1}(0, L)$, i.e., $\bar{u}$ is a classical solution on the entire interval $[0, L]$ of the eigenvalue problem

$$
\begin{gathered}
-u^{\prime \prime}+q(x) u=\bar{\lambda} u \quad \text { in }(0, L) \\
-u^{\prime}(0)=\sigma_{1} \bar{\lambda} u(0), \quad u^{\prime}(L)=\sigma_{2} \bar{\lambda} u(L) .
\end{gathered}
$$

The same is true for $|\bar{u}|$, which is also a minimizer for $\bar{\lambda}$. Hence $\bar{u}\left(x_{0}\right)=\bar{u}^{\prime}\left(x_{0}\right)=0$ and the same holds at $x_{1}$. Thus $\bar{u} \equiv 0$, which is impossible. Hence we have shown that every extremal function for $\bar{\lambda}$ has exactly one zero in $[0, L]$. The same holds for minimizers of $\underline{\lambda}$.

It remains to show the estimates $\lambda_{1}<\bar{\lambda}<\lambda_{2}$ and $\lambda_{-2}<\underline{\lambda}<\lambda_{-1}$, provided $\underline{\lambda}$ is finite. Let us show the inequalities for $\bar{\lambda}$. The inequalities for $\underline{\lambda}$ follow similarly. First, it is clear that $\lambda_{1} \leq \bar{\lambda}$. Since every minimizer for $\bar{\lambda}$ has a zero, whereas the minimizers for $\lambda_{1}$ have no zero, it follows that $\lambda_{1}<\bar{\lambda}$. Likewise, since the second eigenfunction $\varphi_{2}$ has 
a zero we see immediately that $\bar{\lambda} \leq \lambda_{2}$. Let us suppose for contradiction that $\bar{\lambda}=\lambda_{2}$. Testing the equation for $\varphi_{2}$ with $\varphi_{2}^{+}$we obtain

$$
\int_{0}^{L}\left(\left(\varphi_{2}^{+\prime}\right)^{2}+q(x)\left(\varphi_{2}^{+}\right)^{2}\right) d x=\lambda_{2} a\left(\varphi_{2}^{+}, \varphi_{2}^{+}\right)
$$

and since $\bar{\lambda}=\lambda_{2}$ and $\varphi_{2}^{+}$has at least one zero (in fact infinitely many) in $[0, L]$ we find that $\varphi_{2}^{+}$is a minimizer for $\bar{\lambda}$, so it has a unique zero. This contradiction finishes the proof.

Lemma 9. Let $0 \leq q \in L^{\infty}(0, L)$ with $\int_{0}^{L} q d x>0$. Then $\bar{\lambda}=\min \left\{\lambda_{1}^{L}, \lambda_{1}^{0}\right\}$ and $\underline{\lambda}=\max \left\{\lambda_{-1}^{L}, \lambda_{-1}^{0}\right\}$.

Proof. The claim follows if we show that minimizers $\bar{u}, \underline{u}$ for $\bar{\lambda}, \underline{\lambda}$ have no zero in $(0, L)$. Let us show this for $\underline{u}$. Suppose for contradiction that $\underline{u}\left(y_{0}\right)=0$ for some $y_{0} \in(0, L)$. Then $\underline{u}$ is a piecewise $W^{2, \infty}$-solution of

$$
\begin{gathered}
-u^{\prime \prime}+q(x) u=\underline{\lambda} u \quad \text { in }\left(0, y_{0}\right) \cup\left(y_{0}, L\right), \\
-u^{\prime}(0)=\sigma_{1} \underline{\lambda} u(0), \quad u\left(y_{0}\right)=0, \quad u^{\prime}(L)=\sigma_{2} \bar{\lambda} u(L) .
\end{gathered}
$$

By rescaling $\underline{u}$ on $\left[0, y_{0}\right]$ appropriately we can achieve that the rescaled function $\underline{u}$ is a $C^{1}$-function on the entire interval $[0, L]$. The differential equation then implies that in fact $\underline{u}$ is a $W^{2, \infty}$-function on $[0, L]$ solving the above equation pointwise a.e. on $(0, L)$. Hence $\underline{u}$ must be an eigenfunction, but this is impossible since $\lambda_{-2}<\underline{\lambda}<\lambda_{-1}$.

Proof of Theorem 7. Case 1: Let $u$ be a solution of (4.1)-4.2 with $\lambda \in\left(\lambda_{1}, \bar{\lambda}\right]$ and $0 \leq f \in L^{1}(0, L)$ and $g_{1}, g_{2} \geq 0$. By Theorem 2 (b) the solution $u$ cannot be $\geq 0$, i.e., $u^{-} \not \equiv 0$. Testing 4.1 -4.2 with $u^{-}$one obtains

$$
\int_{0}^{L}\left(\left(u^{-\prime}\right)^{2}+q(x)\left(u^{-}\right)^{2}\right) d x=\lambda a\left(u^{-}, u^{-}\right)-\int_{0}^{L} u^{-} f d x-u^{-}(0) g_{1}-u^{-}(L) g_{2} .
$$

By the assumptions on $f, g_{1}, g_{2}$ this implies $\int_{0}^{L}\left(\left(u^{-\prime}\right)^{2}+q(x)\left(u^{-}\right)^{2}\right) d x \leq \lambda a\left(u^{-}, u^{-}\right)$.

Assume for contradiction that $u^{-}$has a zero in $[0, L]$. Then $u^{-}$would be admissible in the variational characterization of $\bar{\lambda}$ and $\bar{\lambda} \leq \lambda$ would follow. By the assumption on $\lambda$ this is only possible for $\lambda=\bar{\lambda}$. Then $u^{-}$is a minimizer for $\bar{\lambda}$ and thus $u^{-}$has exactly one zero. Moreover,

$$
0=\int_{0}^{L} u^{-} f d x+u^{-}(0) g_{1}+u^{-}(L) g_{2} .
$$

However, since either $f \not \equiv 0$ or $g_{1}, g_{2}>0$ the last relation is impossible for a function with only one zero. This contradiction shows that $u<0$ in $[0, L]$.

It remains to prove that the uniform anti-maximum principle does not hold for any $\lambda>\bar{\lambda}$. Assume that it does for such a $\lambda$. Let $\bar{u} \geq 0$ be a minimizer for $\bar{\lambda}$ and define 
$w_{\epsilon}=(\bar{u}-\epsilon)^{+}$for $\epsilon>0$. Then $w_{\epsilon} \rightarrow \bar{u}$ in $H^{1}(0, L)$ as $\epsilon \rightarrow 0$. We may choose $\epsilon$ so small that

$$
\bar{\lambda}<\frac{\int_{0}^{L}\left(w_{\epsilon}^{\prime 2}+q(x) w_{\epsilon}^{2}\right) d x}{a\left(w_{\epsilon}, w_{\epsilon}\right)}<\lambda
$$

and $a\left(w_{\epsilon}, w_{\epsilon}\right) \rightarrow 1$ as $\epsilon \rightarrow 0$. Next we define $0 \leq f \in L^{1}(0, L)$ and $g_{1}, g_{2} \geq 0$ in the following way: let supp $f \cap \operatorname{supp} w_{\epsilon}=\emptyset$. If $0 \in \operatorname{supp} w_{\epsilon}$ then let $g_{1}=0$ and $g_{2}>0$. If $L \in \operatorname{supp} w_{\epsilon}$ then let $g_{2}=0$ and $g_{1}>0$. Note that since $\bar{u}$ has a unique zero at either 0 or $L$ the support of $w_{\epsilon}$ cannot contain both 0 and $L$. Assume now that for the given choice of $f$ and $g$ there is a solution $u$ of (4.1)-(4.2) such that $u<0$ in $[0, L]$. In this case $u$ can be written as $u=-e^{-v}$ with a function $v \in H^{1}(0, L)$. Taking $e^{v} w_{\epsilon}^{2}$ as a test function for 4.1. 4.2) we obtain

$$
\begin{aligned}
\int_{0}^{L}\left(v^{\prime} w_{\epsilon}+w_{\epsilon}^{\prime}\right)^{2} d x-\int_{0}^{L}\left(\left(w_{\epsilon}^{\prime}\right)^{2}+q(x) w_{\epsilon}^{2}\right) d x \\
=-\lambda \underbrace{a\left(w_{\epsilon}, w_{\epsilon}\right)}_{>0}+\int_{0}^{L} f w_{\epsilon}^{2} e^{v} d x+\left.g_{1} e^{v} w_{\epsilon}^{2}\right|_{x=0}+\left.g_{2} e^{v} w_{\epsilon}^{2}\right|_{x=L} .
\end{aligned}
$$

By the assumption on $f, g_{1}, g_{2}$ and $w_{\epsilon}$ the expression involving the product of $f, g_{1}, g_{2}$ with $w_{\epsilon}^{2}$ vanish. Thus

which contradicts 4.3 .

$$
\lambda \leq \frac{\int_{0}^{L}\left(\left(w_{\epsilon}^{\prime}\right)^{2}+q(x) w_{\epsilon}^{2}\right) d x}{a\left(w_{\epsilon}, w_{\epsilon}\right)},
$$

Case 2: For $\lambda \in\left[\underline{\lambda}, \lambda_{-1}\right)$ the argument is analogous. Since $u$ cannot be $\geq 0$, testing with $u^{-}$leads to $\int_{0}^{L}\left(\left(u^{-\prime}\right)^{2}+q(x)\left(u^{-}\right)^{2}\right) d x \leq \lambda a\left(u^{-}, u^{-}\right)$. The assumption that $u^{-}$has a zero leads to $\underline{\lambda} \geq \lambda$, which is only possible if $\underline{\lambda}=\lambda$. This is excluded as above. The optimality proof for the interval $\left[\underline{\lambda}, \lambda_{-1}\right.$ ) follows the same lines as in Case 1.

\begin{tabular}{|c|c|c|c|}
\hline \multirow{2}{*}{ Conditions on $\sigma$} & \multicolumn{3}{|c|}{ Negative eigenvalues } \\
\hline & for $4.1 p-4.2$ & for $\left(D_{L}\right)$ & for $\left(D_{0}\right)$ \\
\hline$\sigma_{1}, \sigma_{2}<0$ & $\begin{array}{ll}\bar{\sigma}>\sigma_{0}: & \lambda_{-2}<\lambda_{-1} \\
\bar{\sigma} \leq \sigma_{0}: & \lambda_{-1}\end{array}$ & $\lambda_{-1}^{L}$ & $\lambda_{-1}^{0}$ \\
\hline$\sigma_{1}<0 \leq \sigma_{2}$ & $\begin{array}{ll}\bar{\sigma}>\sigma_{0}: & \lambda_{-1} \\
\bar{\sigma} \leq \sigma_{0}: & \text { no neg. ev }\end{array}$ & $\lambda_{-1}^{L}$ & no neg. ev \\
\hline $0 \leq \sigma_{1}, \sigma_{2}$ & no neg. ev & no neg. ev & no neg. ev \\
\hline
\end{tabular}

\subsection{The case $q(x) \equiv 0$}

Again we recall from Bandle and Reichel [3] the picture of the existence of negative eigenvalues:

As before, the missing negative eigenvalues are defined as $-\infty$. 
Theorem 10. Let $q \equiv 0$. For $\lambda$ assume the following:

(i) if $\bar{\sigma} \in\left(-\infty, \sigma_{0}\right)$ then $\lambda \in\left[\max \left\{\lambda_{-1}^{L}, \lambda_{-1}^{0}\right\}, 0\right) \cup\left(\lambda_{1}, \min \left\{\lambda_{1}^{L}, \lambda_{1}^{0}\right\}\right]$,

(ii) if $\bar{\sigma} \in\left(\sigma_{0}, \infty\right)$ then $\lambda \in\left[\max \left\{\lambda_{-1}^{L}, \lambda_{-1}^{0}\right\}, \lambda_{-1}\right) \cup\left(0, \min \left\{\lambda_{1}^{L}, \lambda_{1}^{0}\right\}\right]$,

(iii) if $\bar{\sigma}=\sigma_{0}$ then $\lambda \in\left[\max \left\{\lambda_{-1}^{L}, \lambda_{-1}^{0}\right\}, 0\right) \cup\left(0, \min \left\{\lambda_{1}^{L}, \lambda_{1}^{0}\right\}\right]$.

If $0 \leq f \in L^{1}(0, L)$ and $g_{1}, g_{2} \geq 0$ and additionally $f \not \equiv 0$ or $g_{1}, g_{2}>0$ then the solution $u$ of (4.1)-4.2) satisfies $u<0$ in $[0, L]$. Moreover, the above $\lambda$-intervals are optimal for the uniform anti-maximum principle.

Proof. The proof is similar to the proof of Theorem 7 . Let us sketch where the differences occur. First, the values $\bar{\lambda}$ and $\underline{\lambda}$ are defined exactly as in Lemma 8 . The value $\bar{\lambda}$ is always finite, and $\underline{\lambda}$ is finite if at least one of the two values $\sigma_{1}, \sigma_{2}$ is negative. Both values are attained if they are finite, since in the space of $H^{1}$-functions with at least one zero in $[0, L]$ the expression $\left(\int_{0}^{L} v^{\prime 2} d x\right)^{1 / 2}$ is an equivalent norm. Next, one needs to show the following estimates for $\underline{\lambda}, \bar{\lambda}$ :

$$
\begin{aligned}
& \text { Case (i): } \bar{\sigma} \in\left(-\infty, \sigma_{0}\right) \Rightarrow \lambda_{-1}<\underline{\lambda}<0, \quad \lambda_{1}<\bar{\lambda}<\lambda_{2}, \\
& \text { Case (ii): } \bar{\sigma} \in\left(\sigma_{0}, \infty\right) \Rightarrow \lambda_{-2}<\underline{\lambda}<\lambda_{-1}, 0<\bar{\lambda}<\lambda_{1} \text {, } \\
& \text { Case (iii): } \bar{\sigma}=\sigma_{0} \quad \Rightarrow \lambda_{-1}<\underline{\lambda}<0, \quad 0<\bar{\lambda}<\lambda_{1} \text {. }
\end{aligned}
$$

With theses estimates at hand the proofs of the remaining statements of Lemma 8, Lemma 9 and Theorem 10 are exactly the same as before. The variational characterization of $\lambda_{1}, \lambda_{-1}$ (cf. beginning of Subsection 2.2) is valid in the space of $H^{1}(0, L)$ functions with $a(v, 1)=0$, whereas the characterization of $\underline{\lambda}, \bar{\lambda}$ is valid in $H^{1}(0, L)$ only. Thus, for $v \in H^{1}(0, L)$ let us define

$$
w=v-P v=v-\frac{\int_{0}^{L} v d x+\sigma_{1} v(0)+\sigma_{2} v(L)}{L+2 \bar{\sigma}} .
$$

Thus $a(w, 1)=0$ and clearly $\int_{0}^{L} v^{\prime 2} d x=\int_{0}^{L} w^{\prime 2} d x$. Moreover

$$
\begin{aligned}
a(w, w) & =\int_{0}^{L} w^{2} d x+\sigma_{1} w(0)^{2}+\sigma_{2} w(L)^{2} \\
& =\int_{0}^{L} v^{2} d x+\sigma_{1} v(0)^{2}+\sigma_{2} v(L)^{2}-(P v)^{2}(L+2 \bar{\sigma}) \\
& =a(v, v)-(P v)^{2}(L+2 \bar{\sigma}) .
\end{aligned}
$$

Let us start with the estimates in case (i). In this case $a(w, w) \geq a(v, v)$. Hence $\underline{\lambda} \leq \lambda_{-1}$. The estimate $\underline{\lambda} \geq \lambda_{-2}$ follows from the fact that $\varphi_{-2}$ changes sign and can be inserted into the variational characterization of $\underline{\lambda}$. Moreover it follows as in Lemma 8 that $\underline{\lambda}$ cannot be equal to either of the two endpoints. The estimate $0<\bar{\lambda}<\lambda_{1}$ is immediate ( $\varphi_{1}$ is sign-changing and can be inserted into the variational characterization for $\bar{\lambda}$ ).

In case (ii) we find that $a(w, w) \leq a(v, v)$. This is the basis for the estimate $\bar{\lambda} \leq \lambda_{1}$. The rest of the estimates in this case is similar to case (i). 
In the remaining case (iii) we find $a(w, w)=a(v, v)$. Since $\varphi_{-1}$ and $\varphi_{1}$ are signchanging we obtain immediately $\lambda_{-1} \leq \underline{\lambda}$ and $\bar{\lambda} \leq \lambda_{1}$, where equality is excluded as before. It remains to show that $\underline{\lambda}, \bar{\lambda} \neq 0$, which follows from the fact that $\underline{\lambda}=0$ or $\bar{\lambda}=0$ would imply that minimizers are constants, but this is incompatible with having a zero. This completes the proof of the theorem.

\subsection{Examples for constant $q$}

In the case where $\sigma_{1}=\sigma_{2}=\sigma$ and $q \geq 0$ is a constant one can determine the regions of the positivity principle and the anti-maximum principle (almost) explicitly. The solution to the differential equation $-\varphi^{\prime \prime}+q \varphi=\lambda \varphi$ in $(0, L)$ is

$$
\varphi(x)= \begin{cases}A \cos (\sqrt{\lambda-q} x)+B \sin (\sqrt{\lambda-q} x) & \text { if } \lambda>q, \\ A \cosh (\sqrt{q-\lambda} x)+B \sinh (\sqrt{q-\lambda} x) & \text { if } \lambda<q, \\ A x+B & \text { if } \lambda=q .\end{cases}
$$

Case $q>0$ : The eigenvalues $\lambda_{-1}, \lambda_{1}$ are given as the intersection of transcendental functions as follows (cf. [2]). Let $\lambda^{*}$ be the negative root of $\sigma^{2} \lambda^{2}+\lambda-q=0$. Then

$$
\begin{aligned}
& \lambda_{-1}: \quad \tanh (\sqrt{q-\lambda} L / 2)=\sigma \lambda / \sqrt{q-\lambda}, \quad \lambda \in\left(\lambda^{*}, 0\right), \\
& \lambda_{1}: \begin{cases}\tan (\sqrt{\lambda-q} L / 2)=-\sigma \lambda / \sqrt{\lambda-q}, & \sigma<0, \\
q, & \sigma=0, \\
\tanh (\sqrt{q-\lambda} L / 2)=\sigma \lambda / \sqrt{q-\lambda}, & \sigma>0 .\end{cases}
\end{aligned}
$$

Likewise the eigenvalues $\lambda_{-1}^{L}=\lambda_{-1}^{0}$ and $\lambda_{1}^{L}=\lambda_{1}^{0}$ are given by

$$
\begin{aligned}
& \lambda_{-1}^{0}=\lambda_{-1}^{L}: \quad \operatorname{coth}(\sqrt{q-\lambda} L)=\sigma \lambda / \sqrt{q-\lambda}, \\
& \lambda_{1}^{0}=\lambda_{1}^{L}: \quad \begin{cases}\cot (\sqrt{\lambda-q} L)=\lambda \sigma / \sqrt{\lambda-q}, & \sigma<1 /(L q), \\
q, & \sigma=1 /(L q), \\
\operatorname{coth}(\sqrt{q-\lambda} L)=\lambda \sigma / \sqrt{q-\lambda}, & \sigma>1 /(L q) .\end{cases}
\end{aligned}
$$

The results produced by MAPLE are plotted in Figure 2

Case $q=0$ : Although the complete eigenvalue picture is more complicated, the determination is much simpler because according to Theorem 10 we only need to find $\lambda_{-1}$ for $\sigma \geq \sigma_{0}$ and $\lambda_{1}$ for $\sigma \leq \sigma_{0}$.

$$
\begin{aligned}
& \lambda_{-1}: \tanh (\sqrt{-\lambda} L / 2)=-\sigma \sqrt{-\lambda}, \quad \lambda \in\left(\lambda^{*}, 0\right), \quad \text { if } \sigma \geq \sigma_{0}, \\
& \lambda_{1}: \quad \tan (\sqrt{\lambda} L / 2)=-\sigma \sqrt{\lambda} \quad \text { if } \sigma \leq \sigma_{0} .
\end{aligned}
$$

Likewise the eigenvalues $\lambda_{-1}^{L}=\lambda_{-1}^{0}$ and $\lambda_{1}^{L}=\lambda_{1}^{0}$ are given by

$$
\begin{array}{ll}
\lambda_{-1}^{0}=\lambda_{-1}^{L}: \operatorname{coth}(\sqrt{-\lambda} L)=-\sigma \sqrt{-\lambda}, \\
\lambda_{1}^{0}=\lambda_{1}^{L}: \quad \cot (\sqrt{\lambda} L)=\sigma \sqrt{\lambda} .
\end{array}
$$

The results are plotted in Figure 3 . 


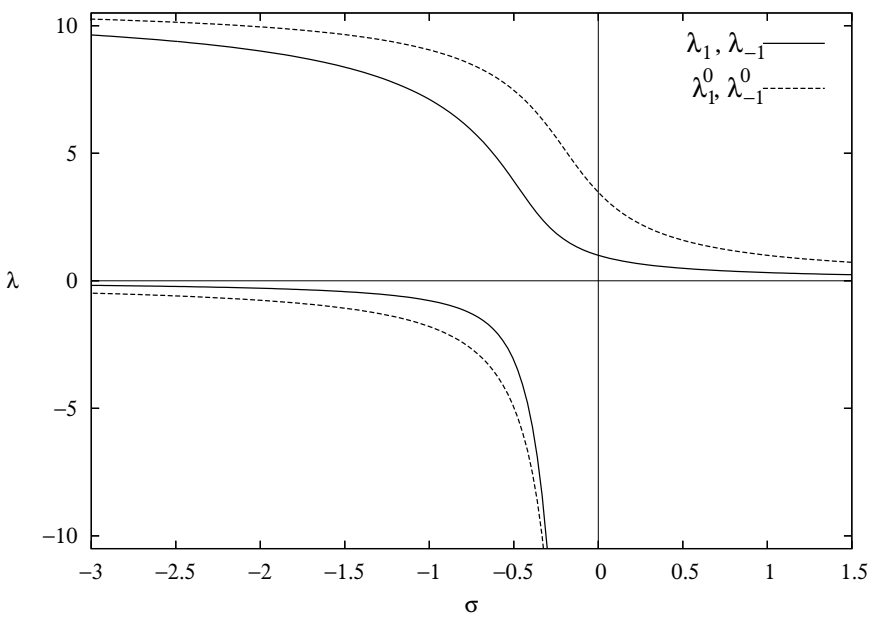

Fig. 2. $q=1, L=1$

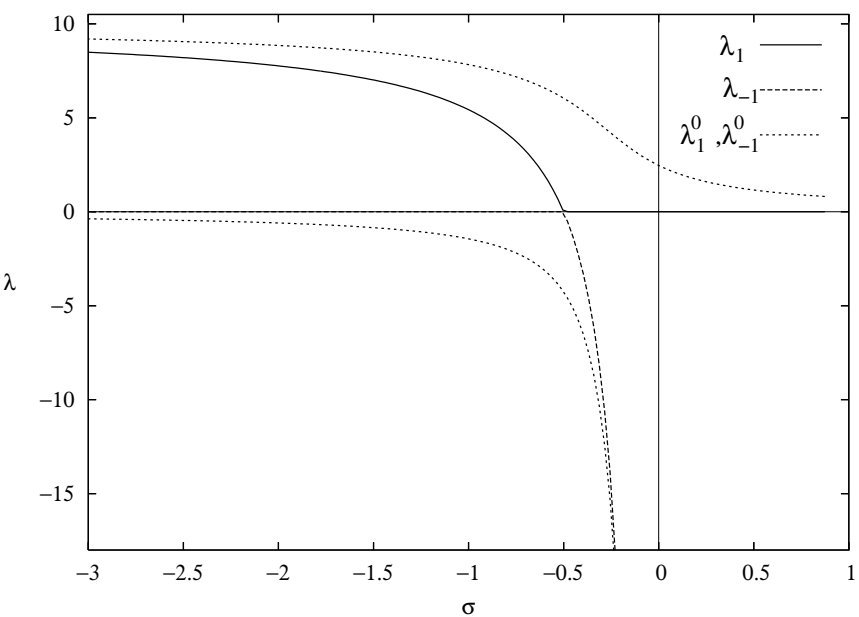

Fig. 3. $q=0, L=1$

\section{Positivity regions for parameter dependent inhomogeneous boundary value} problems

In this section we consider the boundary value problem

$$
-\Delta u+q(x) u=\alpha u+f \quad \text { in } D, \quad u_{n}=\beta u+g \quad \text { on } \partial D, \quad \alpha, \beta \in \mathbb{R} .
$$

We shall use the previous results on the $\lambda$-dependent boundary value problem 1.1 to determine the parameter region for $(\alpha, \beta) \in \mathbb{R}^{2}$ for which the positivity principle holds. 
For this purpose we start with some auxiliary results concerning the $\sigma$-dependence of the smallest positive eigenvalue $\lambda_{1}(\sigma)$ and the largest negative eigenvalue $\lambda_{-1}(\sigma)$ of $(1.2)$.

Without loss of generality (by shifting $\alpha$ if necessary) we may assume that $q(x) \geq$ $q_{0}>0$. Then

$$
\|v\|=\left(\int_{D}\left(|\nabla v|^{2}+q(x) v^{2}\right) d x\right)^{1 / 2}
$$

generates a norm in $H^{1}(D)$ which is equivalent to the standard norm. Denote by $\lambda_{1}^{D}$ the smallest Dirichlet eigenvalue of

$$
-\Delta \varphi+q(x) \varphi=\lambda \varphi \quad \text { in } D, \quad \varphi=0 \quad \text { on } \partial D
$$

and by $\lambda_{1}^{\text {St }}$ the smallest Steklov eigenvalue of the problem

$$
-\Delta \varphi+q(x) \varphi=0 \quad \text { in } D, \quad \varphi_{n}=\lambda \varphi \quad \text { on } \partial D .
$$

Lemma 11. (i) The function $\sigma \mapsto \lambda_{1}(\sigma)$ is continuous and strictly decreasing for $\sigma \in \mathbb{R}$. Moreover

$$
\lim _{\sigma \rightarrow-\infty} \lambda_{1}(\sigma)=\lambda_{1}^{D}, \quad \lim _{\sigma \rightarrow \infty} \lambda_{1}(\sigma)=0 .
$$

(ii) Similarly the function $\sigma \mapsto \lambda_{-1}(\sigma)$ is continuous and strictly decreasing for $\sigma \in(-\infty, 0)$ and

$$
\lim _{\sigma \rightarrow-\infty} \lambda_{-1}(\sigma)=0, \quad \lim _{\sigma \rightarrow 0-} \lambda_{-1}(\sigma)=-\infty .
$$

Proof. Let $J_{\sigma}[v]=\int_{D} v^{2} d x+\sigma \oint_{\partial D} v^{2} d s$ for $v \in H^{1}(D)$. We have the variational characterizations

$$
\frac{1}{\lambda_{1}(\sigma)}=\sup \left\{J_{\sigma}[v]:\|v\|=1\right\}, \quad \frac{1}{\lambda_{-1}(\sigma)}=\inf \left\{J_{\sigma}[v]:\|v\|=1\right\} .
$$

Let $\varphi_{\sigma}$ be the eigenfunction corresponding to $\lambda_{1}(\sigma)$. We shall assume that $\left\|\varphi_{\sigma}\right\|=1$. Moreover, there exists a positive constant $c$ independent of $\sigma$ such that

$$
0<\oint_{\partial D} \varphi_{\sigma}^{2} d s \leq c\left\|\varphi_{\sigma}\right\|^{2}=c,
$$

where the second inequality follows from the trace inequality and the first is a property of eigenfunctions of constant sign (cf. [2], [3]). The variational characterization of $\lambda_{1}(\sigma)$ implies

$$
\begin{aligned}
\frac{1}{\lambda_{1}(\tau)}+(\sigma-\tau) \oint_{\partial D} \varphi_{\sigma}^{2} d s & \geq J_{\tau}\left[\varphi_{\sigma}\right]+(\sigma-\tau) \oint_{\partial D} \varphi_{\sigma}^{2} d s=\frac{1}{\lambda_{1}(\sigma)} \\
& \geq J_{\sigma}\left[\varphi_{\tau}\right]=J_{\tau}\left[\varphi_{\tau}\right]+(\sigma-\tau) \oint_{\partial D} \varphi_{\tau}^{2} d s \\
& =\frac{1}{\lambda_{1}(\tau)}+(\sigma-\tau) \oint_{\partial D} \varphi_{\tau}^{2} d s .
\end{aligned}
$$

Letting $\sigma \rightarrow \tau$ in 5.6 and using the boundedness of the traces from (5.5) we obtain $\lim _{\sigma \rightarrow \tau} \lambda_{1}(\sigma)=\lambda_{1}(\tau)$. For $\sigma>\tau$ the strict monotonicity also follows from (5.6) and from the strict positivity of the boundary integrals as stated in 5.5. 
By introducing the eigenfunction corresponding to $\lambda_{1}^{D}$ as a test function in (5.4) we obtain $\lambda_{1}(\sigma) \leq \lambda_{1}^{D}$ and consequently

$$
\lim _{\sigma \rightarrow-\infty} \lambda_{1}(\sigma)=\alpha \leq \lambda_{1}^{D}
$$

For $\sigma<0$ one gets

$$
1=\lambda_{1}(\sigma)\left(\int_{D} \varphi_{\sigma}^{2} d x+\sigma \oint_{\partial D} \varphi_{\sigma}^{2} d s\right) \leq \lambda_{1}^{D} \int_{D} \varphi_{\sigma}^{2} d x
$$

Since $\left\|\varphi_{\sigma}\right\|=1$ there exists a subsequence $\left\{\varphi_{\sigma_{k}}\right\}_{k=1}^{\infty}, \sigma_{k} \rightarrow-\infty$, which converges to $\tilde{\varphi}$ weakly in $H^{1}(D)$, strongly in $L^{2}(D)$ and in $L^{2}(\partial D)$. Due to 5.7 we have $\tilde{\varphi} \neq 0$. In the weak form of the eigenvalue problem (1.2),

$$
\int_{D}\left(\nabla \varphi_{\sigma_{k}} \cdot \nabla h+q(x) \varphi_{\sigma_{k}} h\right) d x=\lambda_{1}\left(\sigma_{k}\right)\left(\int_{D} \varphi_{\sigma_{k}} h d x+\sigma_{k} \oint_{\partial D} \varphi_{\sigma_{k}} h d s\right)
$$

for all $h \in H^{1}(D)$, we can let $k$ tend to $\infty$. Since the left-hand side and the first term on the right-hand side are bounded we get

$$
\oint_{\partial D} \tilde{\varphi} h d s=\lim _{k \rightarrow \infty} \oint_{\partial D} \varphi_{\sigma_{k}} h d s=0 \quad \text { for all } h \in L^{2}(\partial D) .
$$

Hence trace $\tilde{\varphi}=0$. By taking $h \in H_{0}^{1}(D)$ in 5.8 we see that $\tilde{\varphi}$ is a non-trivial Dirichlet eigenfunction with constant sign and with eigenvalue $\alpha$. Hence $\alpha=\lambda_{1}^{D}$. The last assertion of (i) follows immediately from (5.6).

The continuity and monotonicity proof of the second part (ii) is very similar and will therefore be omitted. To find the limit of $\lambda_{-1}(\sigma)$ as $\sigma \rightarrow-\infty$ take the function $v=1 / \sqrt{\int_{D} q(x) d x}$ as a test function in 5.4). This shows that

$$
J_{\sigma}[v]=(|D|+\sigma|\partial D|) / \int_{D} q(x) d x \rightarrow-\infty \quad \text { as } \sigma \rightarrow-\infty .
$$

Therefore $\lim _{\sigma \rightarrow-\infty} \lambda_{-1}(\sigma)=0$. For the limit $\sigma \rightarrow 0-$ one assumes for contradiction $\lim _{\sigma \rightarrow 0-} \lambda_{-1}(\sigma)=\beta$ for some finite $\beta<0$. Taking convergent subsequences $\varphi_{\sigma_{k}} \rightarrow \tilde{\varphi}$ of eigenfunctions corresponding to $\lambda_{-1}\left(\sigma_{k}\right)$ one finds $0>1 / \beta=\lim _{k \rightarrow \infty} J_{\sigma_{k}}\left[\varphi_{\sigma_{k}}\right]=$ $\int_{D} \tilde{\varphi}^{2} d x \geq 0$. This contradiction shows that $\lim _{\sigma \rightarrow 0-} \lambda_{-1}(\sigma)=-\infty$.

Lemma 12. The functions $\sigma \mapsto \sigma \lambda_{1}(\sigma), \sigma \in \mathbb{R}$, and $\sigma \mapsto \sigma \lambda_{-1}(\sigma), \sigma \in \mathbb{R}^{-}$, are continuous and strictly increasing. In addition we have

$$
\lim _{\sigma \rightarrow-\infty} \sigma \lambda_{1}(\sigma)=-\infty, \quad \lim _{\sigma \rightarrow \infty} \sigma \lambda_{1}(\sigma)=\lambda_{1}^{S t}
$$

and

$$
\lim _{\sigma \rightarrow-\infty} \sigma \lambda_{-1}(\sigma)=\lambda_{1}^{\mathrm{St}}, \quad \lim _{\sigma \rightarrow 0-} \sigma \lambda_{-1}(\sigma)=\infty
$$


Proof. Let $\sigma_{1}<\sigma_{2}$ and let $\phi$ and $\psi$ be the corresponding positive eigenfunctions. Then

$$
\lambda_{1}\left(\sigma_{1}\right)\left(\int_{D} \phi \psi d x+\sigma_{1} \oint_{\partial D} \phi \psi d s\right)=\lambda_{1}\left(\sigma_{2}\right)\left(\int_{D} \phi \psi d x+\sigma_{2} \oint_{\partial D} \phi \psi d s\right) .
$$

Rearranging terms and using the monotonicity of Lemma 11 one finds

$$
\underbrace{\left(\lambda_{1}\left(\sigma_{1}\right)-\lambda_{1}\left(\sigma_{2}\right)\right) \int_{D} \phi \psi d x}_{>0}=\left(\lambda_{1}\left(\sigma_{2}\right) \sigma_{2}-\lambda_{1}\left(\sigma_{1}\right) \sigma_{1}\right) \underbrace{\oint_{\partial D} \phi \psi d s}_{>0} .
$$

The monotonicity of $\sigma \lambda_{1}(\sigma)$ now follows. The same argument applies to $\sigma \lambda_{-1}(\sigma)$. The first statement of 5.9 is obvious. Because of the monotonicity the $\operatorname{limit} \lim _{\sigma \rightarrow \infty} \sigma \lambda_{1}(\sigma)$ exists and equals $\gamma \in(0, \infty]$. The test function $v=1 / \sqrt{\int_{D} q(x) d x}$ yields the estimate $\lambda_{1}(\sigma) \leq \int_{D} q(x) d x /(|D|+\sigma|\partial D|)$. Hence $\gamma=\lim _{\sigma \rightarrow \infty} \sigma \lambda_{1}(\sigma) \leq \int q(x) d x /|\partial D|$, i.e., $\gamma$ is finite. As usual we can consider convergent subsequences of eigenfunctions $\varphi_{\sigma_{k}} \rightarrow \tilde{\varphi}$ with $\sigma_{k} \rightarrow \infty$ as $k \rightarrow \infty$. If we let $k$ tend to $\infty$ in 5.8 and keep in mind that $\lim _{k \rightarrow \infty} \lambda_{1}\left(\sigma_{k}\right)=0$ we see that the limit function $\tilde{\varphi}$ solves

$$
\int_{D}(\nabla \tilde{\varphi} \cdot \nabla h+q(x) \tilde{\varphi} h) d x=\gamma \oint_{\partial D} \tilde{\varphi} h d s \quad \text { for all } h \in H^{1}(D), \quad 1=\gamma \oint_{\partial D} \tilde{\varphi}^{2} d s .
$$

Hence $\tilde{\varphi}$ is non-trivial and (5.11) is the weak form of (5.3). Since $\tilde{\varphi}$ is of constant sign, $\gamma$ is the lowest Steklov eigenvalue, i.e., $\gamma=\lambda_{1}^{\text {St }}$.

The same argument yields $\lim _{\sigma \rightarrow-\infty} \sigma \lambda_{-1}(\sigma)=\lambda_{1}^{\mathrm{St}}$. In order to establish the limit $\sigma \rightarrow 0-$ in 5.10 consider a sequence $\left\{\sigma_{k}\right\}_{k=1}^{\infty}$ such that $\sigma_{k} \rightarrow 0$ - with eigenfunctions $\varphi_{\sigma_{k}}$ corresponding to $\lambda_{-1}\left(\sigma_{k}\right)$. This time let us assume the different normalization $\oint_{\partial D} \varphi_{\sigma_{k}}^{2} d s=1$. We have either

$$
\lim _{\sigma \rightarrow 0-} \sigma \lambda_{-1}(\sigma)=\beta<\infty \quad \text { or } \quad \lim _{\sigma \rightarrow 0-} \sigma \lambda_{-1}(\sigma)=\infty .
$$

Suppose for contradiction that the first case holds. Since $\lambda_{-1}\left(\sigma_{k}\right) \rightarrow-\infty$ we find from the weak form of the eigenvalue equation 5.8 that

$$
\begin{aligned}
\int_{D}\left|\nabla \varphi_{\sigma_{k}}\right|^{2} d x & \leq \int_{D}\left(\left|\nabla \varphi_{\sigma_{k}}\right|^{2}+\left(q(x)-\lambda_{-1}\left(\sigma_{k}\right)\right) \varphi_{\sigma_{k}}^{2}\right) d x \\
& =\lambda_{-1}\left(\sigma_{k}\right) \sigma_{k} \oint_{\partial D} \varphi_{\sigma_{k}}^{2} d s \leq \beta .
\end{aligned}
$$

Note that ||$|v|||:=\left(\int_{D}|\nabla v|^{2} d x+\oint_{\partial D} v^{2} d s\right)^{1 / 2}$ is an equivalent norm in $H^{1}(D)$ and $\left\|\left|\varphi_{\sigma_{k}} \|\right| \leq(1+\beta)^{1 / 2}\right.$. Hence there exists a subsequence, say $\left\{\varphi_{\sigma_{k}}\right\}_{k=1}^{\infty}$, such that $\varphi_{\sigma_{k}} \rightarrow \tilde{\varphi}$ in $H^{1}(D), \varphi_{\sigma_{k}} \rightarrow \tilde{\varphi}$ in $L^{2}(D)$ and in $L^{2}(\partial D)$ as $k \rightarrow \infty$. In particular $\tilde{\varphi} \neq 0$ since $\oint_{\partial D} \tilde{\varphi}^{2} d s=\lim _{k \rightarrow \infty} \oint_{\partial D} \varphi_{\sigma_{k}}^{2} d s=1$. Since $\lambda_{-1}\left(\sigma_{k}\right) \rightarrow-\infty$ as $k \rightarrow \infty$ we see that $\lim _{k \rightarrow \infty} \int_{D} \varphi_{\sigma_{k}}^{2} d x=\int_{D} \tilde{\varphi}^{2} d x=0$ since otherwise we get a contradiction in 5.13. However, we have already seen that $\tilde{\varphi} \neq 0$. This contradiction shows that the second alternative in 5.12) must hold. This completes the proof. 

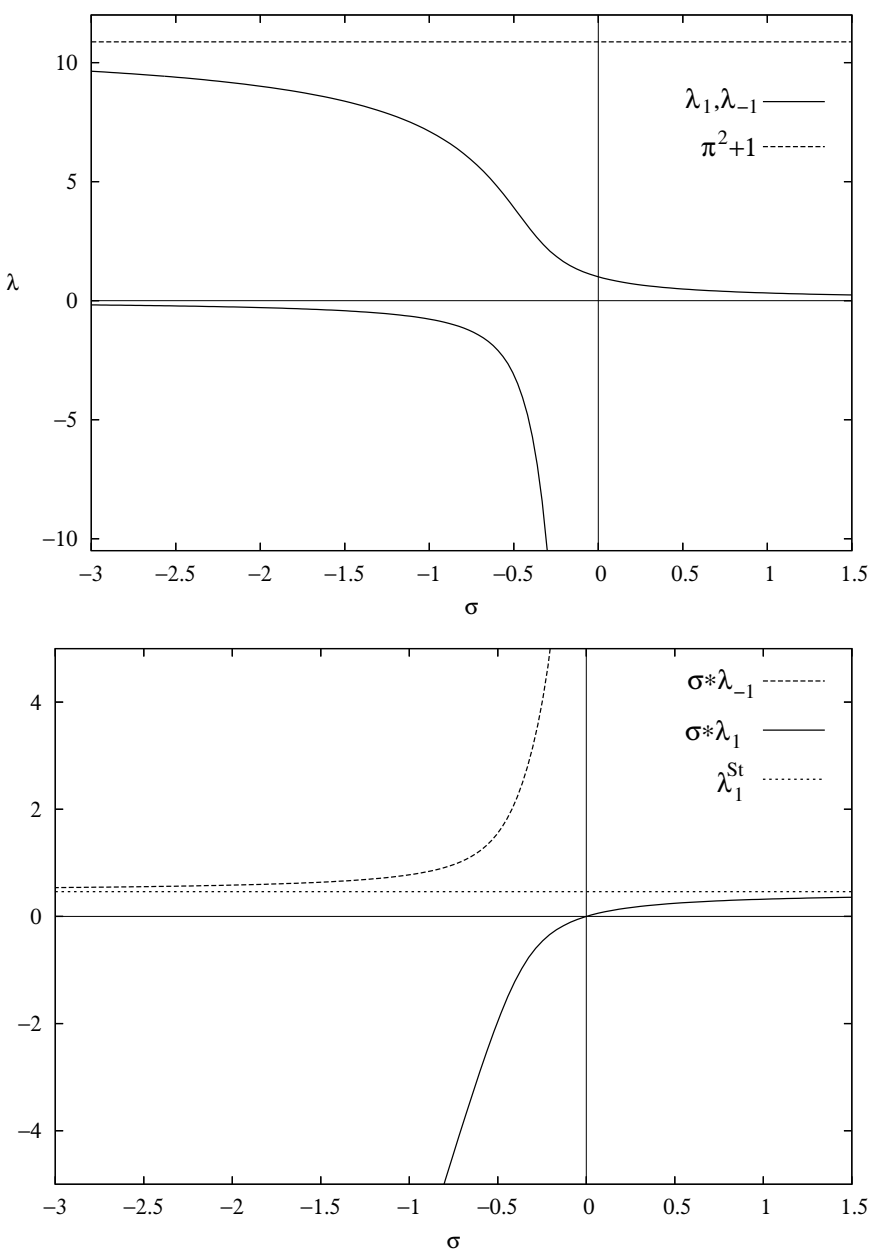

Fig. 4. $q=1, L=1$

For the one-dimensional case with $q=1, D=(0,1)$ the functions $\lambda_{1}(\sigma), \lambda_{-1}(\sigma)$ as well as the functions $\sigma \lambda_{1}(\sigma), \sigma \lambda_{-1}(\sigma)$ are plotted in Figure 4 . Note that in this case $\lambda_{1}^{D}=$ $\pi^{2}+1 \approx 10.8696$ and $\lambda_{1}^{\mathrm{St}}$ is given as the smaller of the two roots of $\lambda^{2}-2 \lambda / \tanh 1+1=0$, $\lambda_{1}^{\text {St }} \approx 0.4621$. Both values are depicted as horizontal lines.

Lemma 13. The function $B:\left(-\infty, \lambda_{1}^{D}\right) \rightarrow \mathbb{R}$ defined by

$$
B(\alpha)= \begin{cases}\alpha \lambda_{1}^{-1}(\alpha) & \text { if } 0<\alpha<\lambda_{1}^{D}, \\ \lambda_{1}^{\mathrm{St}} & \text { if } \alpha=0, \\ \alpha \lambda_{-1}^{-1}(\alpha) & \text { if } \alpha<0,\end{cases}
$$


is continuous, strictly decreasing and satisfies

$$
\lim _{\alpha \rightarrow-\infty} B(\alpha)=\infty, \quad \lim _{\alpha \rightarrow \lambda_{1}^{D}} B(\alpha)=-\infty
$$

Proof. For $\alpha>0$ we express $B(\alpha)$ in terms of $\sigma$, uniquely determined by $\alpha=\lambda_{1}(\sigma)$. Then $B(\alpha)=\sigma \lambda_{1}(\sigma)$. By Lemma $12, \sigma \lambda_{1}(\sigma)$ increases as $\sigma$ increases and $\alpha$ is decreasing in $\sigma$. Therefore $B$ decreases as a function of $\alpha$. By Lemma $11, \alpha \rightarrow \lambda_{1}^{D}$ implies $\sigma \rightarrow-\infty$ and consequently

$$
\lim _{\alpha \rightarrow \lambda_{1}^{D}} B(\alpha)=\lim _{\sigma \rightarrow-\infty} \sigma \lambda_{1}(\sigma)=-\infty .
$$

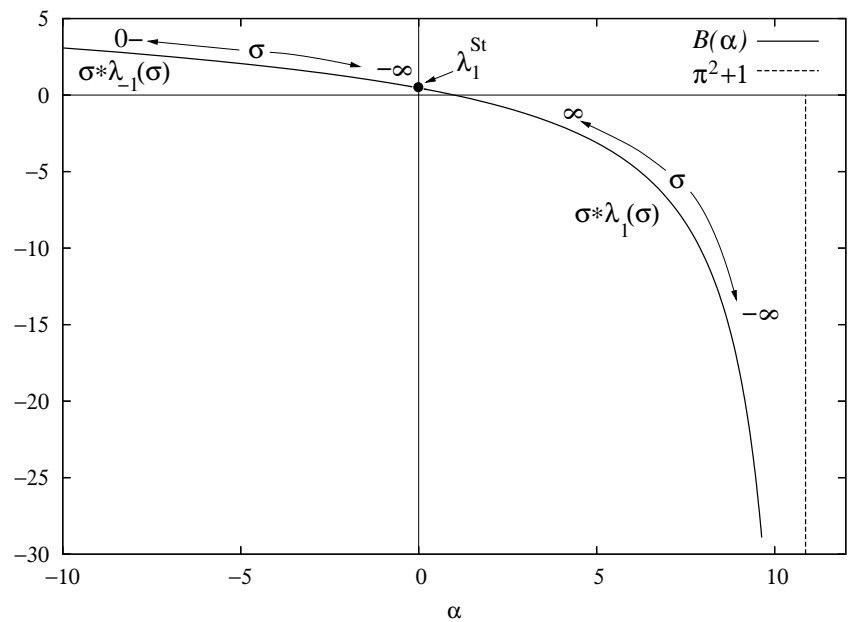

Fig. 5. The function $B(\alpha)$ for $q=1, L=1$

The relation $B(0+)=\lambda_{1}^{\text {St }}$ follows from the fact that $\sigma \rightarrow \infty$ as $\alpha \rightarrow 0$ together with (5.9). Similarly if $\alpha$ is negative we set $\alpha=\lambda_{-1}(\sigma)$. The assertions then follow as before from Lemmas 11 and 12 In particular we have $B(0-)=\lambda_{1}^{\text {St }}$, which shows that $B(\alpha)$ is continuous on the entire interval $\left(-\infty, \lambda_{1}^{D}\right)$.

Theorem 14. Let $0 \leq f \in L^{2}(D)$ and $0 \leq g \in L^{2}(\partial D)$ not both identically zero. Then a solution of 5.1] is positive if and only if $(\alpha, \beta)$ satisfies $\alpha<\lambda_{1}^{D}$ and $\beta<B(\alpha)$.

Proof. Suppose 5.1 has a solution $u>0$ in $\bar{D}$. Then necessarily $\alpha<\lambda_{1}^{D}$, which can be seen as follows. Let $\varphi_{1}^{D}$ be a positive copy of the first Dirichlet eigenfunction. We claim that

$$
\int_{D}\left(\nabla \varphi_{1}^{D} \cdot \nabla \psi+q(x) \varphi_{1}^{D} \psi\right) d x \leq \int_{D} \lambda_{1}^{D} \varphi_{1}^{D} \psi d x \quad \forall \psi \in H^{1}(D) \text { with } \psi \geq 0 \text { in } D \text {. }
$$


This inequality amounts to the weak form of $\partial \varphi_{1}^{D} / \partial n \leq 0$ on $\partial D$. The proof may be folklore or not-we give a short proof in Lemma 18 of the Appendix. Taking $\psi=u$ in (5.14) and using $\varphi_{1}^{D}$ as a test function in the weak form of 5.1] we find

$$
\int_{D}\left(\alpha u \varphi_{1}^{D}+f \varphi_{1}^{D}\right) d x=\int_{D}\left(\nabla u \cdot \nabla \varphi_{1}^{D}+q(x) u \varphi_{1}^{D}\right) d x \leq \int_{D} \lambda_{1}^{D} \varphi_{1}^{D} u d x .
$$

Hence $\alpha \leq \lambda_{1}^{D}$ and if $f \geq 0$, $\not \equiv 0$ then we obtain $\alpha<\lambda_{1}^{D}$. It remains to show that it is impossible to have $\alpha=\lambda_{1}^{D}, f \equiv 0$ and $g \geq 0, \not \equiv 0$. In this case we take the test function $\psi=\left(u-\varphi_{1}^{D}\right)^{-} \in H_{0}^{1}(D)$ both in (5.14) and in the weak form of 5.1) and subtract:

$$
\int_{D}\left(\left|\nabla\left(u-\varphi_{1}^{D}\right)^{-}\right|^{2}+q(x)\left(\left(u-\varphi_{1}^{D}\right)^{-}\right)^{2}\right) d x \leq \lambda_{1}^{D} \int_{D}\left(\left(u-\varphi_{1}^{D}\right)^{-}\right)^{2} d x .
$$

By the variational characterization of $\lambda_{1}^{D}$ we get $\left(u-\varphi_{1}^{D}\right)^{-}=t \varphi_{1}^{D}$ for some $t \geq 0$, i.e., $u=s \varphi_{1}^{D}$ for some $s>0$. But this is impossible since $u>0$ in $\bar{D}$. Thus we know that $\alpha<\lambda_{1}^{D}$. Next we consider the cases $0<\alpha<\lambda_{1}^{D}, \alpha<0$ and $\alpha=0$ separately. that

(i) $0<\alpha<\lambda_{1}^{D}$ : Let $\alpha=\lambda_{1}(\sigma)$ for some $\sigma \in \mathbb{R}$ and $\beta=\tau \alpha$ for some $\tau \in \mathbb{R}$. Note

$$
\begin{aligned}
\beta<B(\alpha) & \Leftrightarrow \tau \alpha<B(\alpha)=\lambda_{1}^{-1}(\alpha) \alpha=\sigma \alpha \\
& \Leftrightarrow \tau<\sigma \\
& \Leftrightarrow \lambda_{1}(\tau)>\alpha .
\end{aligned}
$$

From Theorem 2 and the assumption that either $f$ or $g$ is non-trivial we know that the latter condition is a sharp condition for the existence of positive solutions $u$ of (5.1) with $\beta=\tau \alpha$.

(ii) $\alpha<0$ : We set $\alpha=\lambda_{-1}(\sigma)$ and $\beta=\tau \alpha$. The argument of (i) can be repeated:

$$
\begin{aligned}
\beta<B(\alpha) & \Leftrightarrow \tau \alpha<B(\alpha)=\lambda_{-1}^{-1}(\alpha) \alpha=\sigma \alpha \\
& \Leftrightarrow \tau>\sigma \\
& \Leftrightarrow \lambda_{-1}(\tau)<\alpha,
\end{aligned}
$$

and the latter condition is again sharp by Theorem 2

(iii) $\alpha=0$ : In this case the necessity/sufficiency of the condition $\beta<\lambda_{1}^{\text {St }}=B(\alpha)$ for the existence of positive solutions is well known from the theory of Steklov problems.

\section{Appendix}

Lemma 15. Suppose $D \subset \mathbb{R}^{N}$ is a bounded Lipschitz domain. There exists a constant $\tilde{C}=\tilde{C}(D)$ such that for every $\epsilon \in(0,1)$ we have

$$
\oint_{\partial D} z^{2} d s \leq \frac{\tilde{C}}{\epsilon} \int_{D} z^{2} d x+\tilde{C} \epsilon \int_{D}|\nabla z|^{2} d x \quad \text { for every } z \in H^{1}(D) .
$$


Proof. Let $\xi$ be a smooth vector field in a neighbourhood of $D$ such that $\xi \cdot n \geq c_{0}>0$ a.e. on $\partial D$. For the existence of $\xi$, cf. Lemma 30 in [2]. The inequality

$$
\oint_{\partial D} c_{0} z^{2} d s \leq \int_{D}\left((\operatorname{div} \xi) z^{2}+2 z \xi \cdot \nabla z\right) d x \leq \int_{D} C\left(z^{2}+\frac{1}{\epsilon} z^{2}+\epsilon|\nabla z|^{2}\right) d x
$$

is equivalent to the claim.

Lemma 16. Suppose $D \subset \mathbb{R}^{N}$ is a bounded Lipschitz domain. Let $0 \leq A \in L^{p_{1}}(D)$, $0 \leq B \in L^{p_{2}}(\partial D)$ with $p_{1}>N / 2$ and $p_{2}>N-1$. For $z \in H^{1}(D)$ and $t>0$ the following inequalities hold:

$$
\begin{aligned}
& \int_{D} A(x) z^{2} d x \leq t^{1-2 p_{1} / N}\|A\|_{L^{p_{1}} /(D)}^{2 p_{1} / N} \int_{D}|\nabla z|^{2} d x+t \int_{x \in D: A(x) \leq t} z^{2} d x, \\
& \oint_{\partial D} B(x) z^{2} d s \leq t^{1-p_{2} /(N+1)}\|B\|_{L^{p_{2}(\partial D)}}^{p_{2} /(N-1)} \int_{D}|\nabla z|^{2} d x+t \oint_{x \in \partial D: B(x) \leq t} z^{2} d s .
\end{aligned}
$$

Proof. We give the proof of the first inequality and write $p=p_{1}$ for simplicity. The proof of the second is analogous. Let $D_{t}=\{x \in D: A(x) \geq t\}$. The inequality

$$
t^{N / 2} \operatorname{meas}\left(D_{t}\right) \leq \int_{D_{t}} A(x)^{N / 2} d x \leq\|A\|_{L^{p}(D)}^{N / 2} \operatorname{meas}\left(D_{t}\right)^{1-N /(2 p)}
$$

implies

$$
\operatorname{meas}\left(D_{t}\right) \leq\|A\|_{L^{p}(D)}^{p} t^{-p}, \quad \int_{D_{t}} A(x)^{N / 2} d x \leq\|A\|_{L^{p}(D)}^{p} t^{N / 2-p} .
$$

Hence

$$
\begin{aligned}
\int_{D} A(x) z^{2} d x & \leq \int_{D_{t}} A(x) z^{2} d x+t \int_{D \backslash D_{t}} z^{2} d x \\
& \leq\left(\int_{D_{t}} A(x)^{N / 2} d x\right)^{2 / N}\|z\|_{L^{2 N /(N-2)}(D)}^{2}+t \int_{D \backslash D_{t}} z^{2} d x \\
& \leq\|A\|_{L^{p}(D)}^{2 p / N} t^{1-2 p / N}\|\nabla z\|_{L^{2}(D)}^{2}+t \int_{D \backslash D_{t}} z^{2} d x
\end{aligned}
$$

which implies the claim.

Lemma 17. Suppose $D \subset \mathbb{R}^{N}$ is a bounded Lipschitz domain and let $a \in L^{\infty}(D)$, $b \in L^{\infty}(\partial D)$.

(i) Let $f \in L^{p_{1}}(D)$ and $g \in L^{p_{2}}(\partial D)$ with $p_{1}>N / 2$ and $p_{2}>N-1$. There exists a constant $C=C\left(\|a\|_{\infty},\|b\|_{\infty}, D, N, p_{1}, p_{2}\right)$ such that every weak solution $v \in H^{1}(D)$ of

$$
-\Delta v=a(x) v+f(x) \quad \text { in } D, \quad v_{n}=b(x) v+g(x) \quad \text { on } \partial D
$$

satisfies $\|v\|_{L^{\infty}(D)} \leq C\left(\|v\|_{L^{2}(D)}+\|f\|_{L^{p_{1}(D)}}+\|g\|_{L^{p_{2}(D)}}\right)$. 
(ii) For any $p \in[1, n /(n-2))$ there exists a constant $C=C\left(\|a\|_{\infty},\|b\|_{\infty}, D, N, p\right)$ such that every weak solution $0 \leq v \in H^{1}(D)$ of

$$
-\Delta v \geq a(x) v \quad \text { in } D, \quad v_{n} \geq b(x) v \quad \text { on } \partial D
$$

satisfies $\inf _{D} v(x) \geq C\|v\|_{L^{p}(D)}$. In particular, either $v \equiv 0$ or there exists $\delta>0$ such that $v \geq \delta>0$ a.e. in $D$ and trace $v \geq \delta>0$ a.e. on $\partial D$.

Proof. The proof is based on Moser's iteration method (cf. Gilbarg and Trudinger [9]). let

(i) Let $k=\|f\|_{L^{p_{1}(D)}}+\|g\|_{L^{p_{2}(\partial D)}}$ and define $\bar{v}=v^{+}+k$. For fixed $L>0, s>0$

$$
\varphi=\bar{v} \min \left\{\bar{v}^{2 s}, L^{2 s}\right\}-k^{2 s+1}, \quad w=\bar{v} \min \left\{\bar{v}^{s}, L^{s}\right\} .
$$

Then

$$
\nabla \varphi=\nabla v^{+}\left(\min \left\{\bar{v}^{2 s}, L^{2 s}\right\}+2 s \bar{v}^{2 s} \chi_{\{\bar{v} \leq L\}}\right), \quad \nabla w=\nabla v^{+}\left(\min \left\{\bar{v}^{s}, L^{s}\right\}+s \bar{v}^{s} \chi_{\{\bar{v} \leq L\}}\right),
$$

and hence $|\nabla w|^{2} \leq(s+1) \nabla v \cdot \nabla \varphi$. Taking $\varphi$ as a test function in 5.15) and noting that $\varphi=0$ whenever $v \leq 0$ we obtain

$$
\begin{aligned}
& \frac{1}{s+} \int_{D}|\nabla w|^{2} d x \\
& \quad \leq \int_{D}\left(|a| v^{+}+|f|\right) \varphi d x+\oint_{D}\left(|b| v^{+}+|g|\right) \varphi d s \\
& \quad \leq \int_{D}\left(|a| v^{+}+|f|\right) \bar{v} \min \left\{\bar{v}^{2 s}, L^{2 s}\right\} d x+\oint_{\partial D}\left(|b| v^{+}+|g|\right) \bar{v} \min \left\{\bar{v}^{2 s}, L^{2 s}\right\} d s .
\end{aligned}
$$

By the inequalities $\left(|a| v^{+}+|f|\right) \leq(|a|+|f| / k) \bar{v},\left(|b| v^{+}+|g|\right) \leq(|b|+|g| / k) \bar{v}$ we obtain

$$
\frac{1}{s+1} \int_{D}|\nabla w|^{2} d x \leq \int_{D} A(x) w^{2} d x+\oint_{\partial D} B(x) w^{2} d s,
$$

where $A(x)=|a(x)|+|f(x)| / k$ and $B(x)=|b(x)|+|g(x)| / k$. This choice of $A, B$ implies in particular $\|A\|_{L^{p_{1}(D)}},\|B\|_{L^{p_{2}(\partial D)}} \leq C\left(\|a\|_{\infty},\|b\|_{\infty}\right)$. Here and in the following the same symbol $C$ denotes different constants depending only on $\|a\|_{\infty},\|b\|_{\infty}$, $D, N, p_{1}, p_{2}$. Next we apply Lemma 16 to (5.17) for the volume integral with $t=$ $(2 s+2)^{1 /\left(2 p_{1} / N-1\right)}\|A\|_{L^{p_{1}(D)}}^{\gamma_{1}}, \gamma_{1}=p_{1} /\left(p_{1}-N / 2\right)$ and for the surface integral with $t=(2 s+2)^{1 /\left(p_{2} /(N+1)-1\right)}\|B\|_{L^{p_{2}(\partial D)}}^{\gamma_{2}}, \gamma_{2}=p_{2} /\left(p_{2}-N+1\right)$. Thus we obtain

$$
\int_{D}|\nabla w|^{2} d x \leq C(2 s+2)^{p_{1} /\left(p_{1}-N / 2\right)} \int_{D} w^{2} d x+C(2 s+2)^{p_{2} /\left(p_{2}-(N+1)\right)} \oint_{\partial D} w^{2} d s .
$$

Next we use Lemma 15 with $\epsilon=\frac{1}{2 \tilde{C} C}(2 s+2)^{-p_{2} /\left(p_{2}-(N+1)\right)}$ and deduce from 5.18 that

$$
\int_{D}|\nabla w|^{2} d x \leq 2\left(C(2 s+2)^{p_{1} /\left(p_{1}-N / 2\right)}+2 C \tilde{C}^{2}(2 s+2)^{p_{2} /\left(p_{2}-(N+1)\right)}\right) \int_{D} w^{2} d x
$$


and by adding the square of the $L^{2}$-norm of $w$ to both sides and using the Sobolev inequality we find

$$
\|w\|_{2 N /(N-2)} \leq C(s+1)^{\gamma}\|w\|_{2}, \quad \gamma=\max \left\{\frac{p_{1}}{p_{1}-N / 2}, \frac{p_{2}}{p_{2}-(N+1)}\right\} .
$$

If $w \in L^{2(s+1)}(D)$ we can let $L$ tend to infinity in 5.19 , and obtain $\bar{v} \in L^{(s+1) 2 N /(N-2)}(D)$ and

$$
\|\bar{v}\|_{(s+1) 2 N /(N-2)} \leq(C(s+1))^{\gamma /(s+1)}\|\bar{v}\|_{2(s+1)} .
$$

Hence, if $s_{0}=0$ and $s_{k+1}+1=\left(s_{k}+1\right) \frac{N}{N-2}$ then

$$
\|\bar{v}\|_{2\left(s_{k+1}+1\right)} \leq\left(C\left(s_{k}+1\right)\right)^{\gamma /\left(s_{k}+1\right)}\|\bar{v}\|_{2\left(s_{k}+1\right)} .
$$

Since $s_{k}+1=\left(\frac{N}{N-2}\right)^{k}, k \in \mathbb{N}_{0}$, it follows that

$$
\begin{aligned}
\|\bar{v}\|_{\infty}=\lim _{k \rightarrow \infty}\|\bar{v}\|_{2\left(s_{k+1}+1\right)} & \leq \prod_{k=0}^{\infty}\left(C\left(s_{k}+1\right)\right)^{\gamma /\left(s_{k}+1\right)}\|\bar{v}\|_{2} \\
& =\exp \left(\sum_{k=0}^{\infty} \frac{\gamma \ln C\left(s_{k}+1\right)}{s_{k}+1}\right)\|\bar{v}\|_{2} \\
& \leq C \exp \left(\sum_{k=0}^{\infty} \gamma k\left(\frac{N-2}{N}\right)^{k}\right)\|\bar{v}\|_{2},
\end{aligned}
$$

where the last sum converges. Recalling the definition of $\bar{v}=v^{+}+\|f\|_{L^{p_{1}(D)}}+\|g\|_{L^{p_{2}(\partial D)}}$ we have obtained the upper estimate in statement (i) of the lemma for $v^{+}$. The estimate for $v^{-}$follows from $v^{-}=(-v)^{+}$.

(ii) Now we turn to the lower estimate of the lemma. Let $\varphi=\bar{v}^{s}$ with $s<0$ where $\bar{v}=v+L$ with $L>0$. Then $\nabla v \cdot \nabla \varphi=s \bar{v}^{s-1}|\nabla \bar{v}|^{2}$ and taking $\varphi$ as a test function in 5.16, we find

$$
\begin{aligned}
s \int_{D} \bar{v}^{s-1}|\nabla \bar{v}|^{2} d x & \geq \int_{D} a^{-}(x) \bar{v}^{s+1} d x+\oint_{\partial D} b^{-}(x) \bar{v}^{s+1} d s \\
& \geq-C\left(\int_{D} \bar{v}^{s+1} d x+\oint_{\partial D} \bar{v}^{s+1} d s\right) .
\end{aligned}
$$

If $s \neq-1$ we set $V=\bar{v}^{(s+1) / 2}$ and obtain $|\nabla V|^{2}=\left(\frac{s+1}{2}\right)^{2}|\nabla \bar{v}|^{2} \bar{v}^{s-1}$. If $s=-1$ then we set $V=\log \bar{v}$ and obtain $|\nabla V|^{2}=\bar{v}^{-2}|\nabla \bar{v}|^{2}$. Together with 5.21 this implies

$$
\int_{D}|\nabla V|^{2} d x \leq \begin{cases}C|s+1|\left(\int_{D} V^{2} d x+\oint_{\partial D} V^{2} d s\right) & \text { if } s \neq-1, \\ C & \text { if } s=-1,\end{cases}
$$

with $C=C\left(\|a\|_{\infty},\|b\|_{\infty}\right)$. By Lemma 15 with $\epsilon=1 /(2 C \tilde{C}|s+1|)$ this implies

$$
\int_{D}|\nabla V|^{2} d x \leq C|s+1|^{2} \int_{D} V^{2} d x
$$


provided $|s+1| \geq\left|s_{0}+1\right|>0$. Adding the square of the $L^{2}$-norm of $V$ to both sides and using the Sobolev inequality we get

$$
\|V\|_{2 N /(N-2)} \leq C|s+1|\|V\|_{2} .
$$

For any $p \in \mathbb{R}$ let

$$
\Phi(p)=\left(\int_{D} \bar{v}^{p} d x\right)^{1 / p}
$$

Then 5.23 implies $\Phi\left((s+1) \frac{N}{N-2}\right)^{(s+1) / 2} \leq C|s+1| \Phi(s+1)^{(s+1) / 2}$, i.e.,

$$
\Phi\left((s+1) \frac{N}{N-2}\right) \underset{\leq}{\geq}(C|s+1|)^{-2 /|s+1|} \Phi(s+1) \quad \text { if }\left\{\begin{array}{l}
s<-1 \\
-1<s<0 .
\end{array}\right.
$$

This estimate will be iterated. Set $s_{k+1}+1=\left(s_{k}+1\right) \frac{N}{N-2}$. Then $s_{k}+1=\left(s_{1}+1\right)\left(\frac{N}{N-2}\right)^{k-1}$ and if $s_{1}<-1$ then

$$
\Phi\left(s_{k+1}+1\right) \geq\left(C\left|s_{k}+1\right|\right)^{-2 /\left|s_{k}+1\right|} \Phi\left(s_{k}+1\right) .
$$

Solving this difference inequality we find that

$$
\begin{aligned}
\inf _{D} \bar{v} \geq \lim _{k \rightarrow \infty} \Phi\left(s_{k+1}+1\right) & \geq \prod_{k=1}^{\infty}\left(C\left|s_{k}+1\right|\right)^{-2 /\left|s_{k}+1\right|} \Phi\left(s_{1}+1\right) \\
& =\exp \left(\sum_{k=1}^{\infty} \frac{-2 \ln C\left|s_{k}+1\right|}{\left|s_{k}+1\right|}\right) \Phi\left(s_{1}+1\right) \\
& \geq \frac{C}{\exp \left(\sum_{k=1}^{\infty}(k-1)\left(\frac{N-2}{N}\right)^{k-1}\right)} \Phi\left(s_{1}+1\right),
\end{aligned}
$$

and since the last sum converges we have obtained

$$
\inf _{D} \bar{v} \geq C \Phi\left(s_{1}+1\right)
$$

for some initial number $s_{1}<-1$, which we can still choose. Similarly, if we choose $s_{1} \in(-1,0)$ we can iterate 5.24$)$ as long as $s_{k} \in(-1,0)$ and obtain $\Phi\left(\frac{N}{N-2}\left(s_{k}+1\right)\right) \leq$ $C \Phi\left(s_{k}+1\right) \leq C \Phi\left(s_{1}+1\right)$. In other words, we have

$$
\Phi(p) \leq C \Phi\left(p_{0}\right) \quad \text { whenever } \quad 0<p_{0}<p<\frac{N}{N-2} .
$$

It remains to give a lower bound for $\Phi(s)$ for some $s<0$. For this purpose recall the JohnNirenberg inequality (cf. Gilbarg and Trudinger [9]): suppose $V \in W^{1,1}(D)$ is such that there exists $C>0$ with $\int_{B_{r}}|\nabla V| d x \leq C r^{N-1}$ for every ball $B_{r} \subset D$. Then there exists a number $p_{0}>0$ such that $\int_{D} e^{p_{0}|V-\tilde{V}|} d x<C$ where $\tilde{V}=|D|^{-1} \int_{D} V d x$. We apply this for $V=\log \bar{v}$. Then the second inequality of 5.22) shows that $V \in W^{1,2}(D)$ and hence $\int_{B_{r}}|\nabla V| d x \leq C r^{N / 2}\left(\int_{B_{r}}|\nabla V|^{2} d x\right)^{1 / 2} \leq C r^{N-1}$. The last inequality is obtained 
similarly to (5.22) by testing (5.16) with $\varphi=\bar{v}^{-1} \eta^{2}$ where $\eta$ is a suitable cutoff function in a ball $B_{2 r}$. Thus, the John-Nirenberg inequality applies and together with the trivial estimate $\pm(V-\tilde{V}) \leq|V-\tilde{V}|$ we obtain

$$
\begin{aligned}
& \int_{D} e^{p_{0} V} d x \leq C e^{p_{0} \tilde{V}}, \quad \int_{D} e^{-p_{0} V} d x \leq C e^{-p_{0} \tilde{V}}, \quad \text { i.e. } \\
& \int_{D} e^{p_{0} V} d x \int_{D} e^{-p_{0} V} d x \leq C^{2} .
\end{aligned}
$$

Recalling the definition of $V=\log \bar{v}$ this shows that $\int_{D} \bar{v}^{p_{0}} d x \int_{D} \bar{v}^{-p_{0}} d x \leq C^{2}$ and hence

$$
\left(\int_{D} \bar{v}^{p_{0}} d x\right)^{1 / p_{0}} \leq C^{2 / p_{0}}\left(\int_{D} \bar{v}^{-p_{0}} d x\right)^{-1 / p_{0}}
$$

Together with (5.25) this shows that

$$
\inf _{D} \bar{v} \geq C \Phi\left(-p_{0}\right) \geq C^{\prime} \Phi\left(p_{0}\right) \geq C^{\prime \prime} \Phi(p),
$$

where $p \in\left[1, \frac{N}{N-2}\right)$. The last part of this inequality follows either from Hölder's inequality if $p_{0} \geq \frac{N}{N-2}$ or from 5.26 if $p_{0} \in\left(0, \frac{N}{N-2}\right)$. Letting $L \rightarrow 0$ we obtain the claim of statement (ii) of the lemma.

Lemma 18. Let $D$ be a bounded Lipschitz domain, $0 \leq q \in L^{\infty}(D), 0 \leq h \in L^{2}(D)$ and $0 \leq v \in H_{0}^{1}(D)$ a weak solution of

$$
-\Delta v+q(x) v=h \quad \text { in } D, \quad v=0 \quad \text { on } \partial D .
$$

Then

$$
\int_{D}(\nabla v \cdot \nabla \psi+q(x) v \psi) d x \leq \int_{D} h \psi d x \quad \forall \psi \in H^{1}(D) \text { with } \psi \geq 0 .
$$

Proof. Let us first prove the result for $h \in C^{\infty}(D)$ and $q \in C^{\infty}(D)$. Then $v \in C^{\infty}(D)$ and (5.27) holds pointwise in $D$. By Sard's lemma for almost every $0<s<\|v\|_{\infty}$ the super-level set $D_{s}=\{x \in D: v(x)>s\}$ has a smooth boundary. Thus we obtain for almost every $s \in\left(0,\|v\|_{\infty}\right)$ and every $\psi \in H^{1}(D)$ with $\psi \geq 0$,

$$
\int_{D_{s}}(\nabla v \cdot \nabla \psi+q(x) v \psi) d x=\int_{D_{s}} h \psi d s+\oint_{\partial D_{s}} \psi \underbrace{\partial_{n} v}_{\leq 0} d s \leq \int_{D_{s}} h \psi d s .
$$

Choosing an appropriate sequence $s \rightarrow 0$ we obtain (5.28). For the general case we can approximate $h \in L^{2}(D), q \in L^{\infty}(D)$ by sequences $h_{k}, q_{k} \in C^{\infty}(D)$ with $h_{k} \rightarrow h$ and $q_{k} \rightarrow q$ in $L^{2}(D)$. Let $v_{k} \in H_{0}^{1}(D) \cap C^{\infty}(D)$ be the corresponding solution. Then 5.28 holds for $v_{k}, q_{k}, h_{k}$ and every test function $\psi \in C^{\infty}(D)$ with $\psi \geq 0$. Letting $k \rightarrow \infty$ we retrieve the result for $v, q, h$.

Acknowledgments. Parts of this paper were written when the third author was visiting the Université du Littoral Côte d'Opale in Spring 2005. He expresses his thanks for the hospitality and support. 


\section{References}

[1] Arias, M., Campos, J., Gossez, J.-P.: On the antimaximum principle and the Fučik spectrum of the Neumann $p$-Laplacian. Differential Integral Equations 13, 217-226 (2000) Zbl 0979.35048 MR 1811956

[2] Bandle, C., v. Below, J., Reichel, W.: Parabolic problems with dynamical boundary conditions: eigenvalue expansions and blow up. Rend. Lincei Mat. Appl. 17, 35-67 (2006) MR 2237743

[3] Bandle, C., Reichel, W.: A linear parabolic problem with non-dissipative dynamical boundary conditions. In: Recent Advances in Elliptic and Parabolic Problems (Zurich, 2004), World Sci. (2006)

[4] v. Below, J., François, G.: Spectral asymptotics for the Laplacian under an eigenvalue dependent boundary condition. Bull. Belg. Math. Soc. 12, 505-519 (2005) MR 2205994

[5] Clément, Ph., Peletier, L. A.: An anti-maximum principle for second-order elliptic operators. J. Differential Equations 34, 218-229 (1979) Zbl 0387.35025 MR 0550042

[6] Clément, Ph., Sweers, G.: Uniform anti-maximum principle for polyharmonic boundary value problems. Proc. Amer. Math. Soc. 129, 467-474 (2001) Zbl 0959.35044 MR 1800235

[7] Fleckinger, J., Gossez, J.-P., Takáč, P., de Thélin, F.: Existence, nonexistence et principe de l'antimaximum pour le $p$-laplacien. C. R. Acad. Sci. Paris Sér. I 321, 731-734 (1995) Zbl 0840.35016 MR 1354715

[8] Fleckinger-Pellé, J., Takáč, P.: Maximum and anti-maximum principles for some elliptic problems. In: Advances in Differential Equations and Mathematical Physics (Atlanta, GA, 1997), Contemp. Math. 217, Amer. Math. Soc., Providence, RI, 19-32 (1998) Zbl 0894.35015 MR 1605266

[9] Gilbarg, D., Trudinger, N. S.: Elliptic Partial Differential Equations of Second Order. 3rd ed., Springer (2001) Zbl 1042.35002 MR 1814364

[10] Godoy, T., Gossez, J.-P., Paczka, S.: Antimaximum principle for elliptic problems with weight. Electron. J. Differential Equations 1999, no. 22, 15 pp. Zbl 0920.35045 MR 1689386

[11] Hess, P.: An antimaximum principle for linear elliptic equations with an indefinite weight function. J. Differential Equations 41, 369-374 (1981) MR 0633824

[12] Hess, P.: On positive solutions of semilinear periodic-parabolic problems. In: InfiniteDimensional Systems (Retzhof, 1983), Lecture Notes in Math. 1076, Springer, Berlin, 101114 (1984) Zbl 0556.35066 MR 0763357

[13] Hess, P., Kato, T.: On some linear and nonlinear eigenvalue problems with an indefinite weight function. Comm. Partial Differential Equations 5, 999-1030 (1980) Zbl 0477.35075 MR 0588690

[14] Reichel, W.: Sharp parameter ranges in the uniform anti-maximum principle for second-order ordinary differential operators. Z. Angew. Math. Phys. 54, 822-838 (2003) Zbl 1047.34018 MR 2019184 